\title{
TECHNIQUES FOR ESTIMATING MAGNITUDE AND FREQUENCY OF FLOODS IN MINNESOTA
}

\section{U. S. GEOLOGICAL SURVEY}

Water-Resources Investigations 77-31

Prepared in cooperation with the

Minnesota Department of Transportation, Division of Highways and

Minnesota Department of Natural Resources, Division of Waters

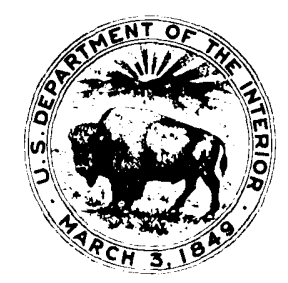

(200)

WRi

no.77-0031 
TECHNIQUES FOR ESTIMATING MAGNITUDE AND FREQUENCY OF FLOODS IN MINNESOTA

\section{Author(s)}

Lowe11 C. Guetzkow

1. Report No.

2.

3. Recipient's Accession No.

5. Report Date

May 1977

6.

8. Performing Organization Rept. No. USGS/WRI 77-31

9. Performing Organization Name and Address

U.S. Geological Survey, Water Resources Division

Room 1033 Post Office Building

St. Paul, Minnesota 55101

12. Sponsoring Organization Name and Address

U.S. Geological Survey, Water Resources Division

Room 1033 Post Office Building

St. Paul, Minnesota 55101
10. Project/Task/Work Unit No.

11. Contract/Grant No.

15. Supplementary Notes Prepared in cooperation with Minnesota Department of Transportation, Division of Highways and Minnesota Department of Natural Resources, Division of Waters.

16. Abstracts Estimating relations have been developed to provide engineers and designers with improved techniques for defining flow-frequency characteristics to satisfy hydraulic planning and design requirements. The magnitude and frequency of floods up to the 100-year recurrence interval can be determined for most streams in Minnesota by methods presented. By multiple regression analysis, equations have been developed for estimating flood-frequency relations at ungaged sites on natural flow streams. Eight distinct hydrologic regions are delineated within the State with boundaries defined generally by river basin divides. Regression equations are provided for each region which relate selected frequency floods to significant basin parameters. For main-stem streams, graphs are presented showing floods for selected recurrence intervals plotted against contributing drainage area. Flow-frequency estimates for intervening sites along the Minnesota River, Mississippi River, and the Red River of the North can be derived from these graphs. Flood-frequency characteristics are tabulated for 201 gaging stations having 10 or more years of record.

17. Key Words and Document Analysis. 17a. Descriptors

* Frequency analysis, * Regression analysis, * Regional analysis,

* Flow characteristics, * Estimating equations, Design flow, Regiona1

flood, Small watersheds, Floods, Hydraulic design, Natural flow, Hydrologic data, Peak discharge, Probability, Statistical methods.

17b. Identifiers/Open-Ended Terms

* Flood frequency, * Minnesota, Recurrence interval, Ungaged watersheds.

17c. COSATI Field/Group

\begin{tabular}{|l|l|l|}
\hline 18. Availability Statement No restriction on distribution. & $\begin{array}{l}\text { 19. Security Class (This } \\
\text { Report) } \\
\text { This report may be purchased from: }\end{array}$ & $\begin{array}{l}\text { 21. No. of Pages } \\
\text { UNCLASSIEJED }\end{array}$ \\
$\begin{array}{l}\text { National Technical Information Service } \\
\text { Springfield. Virginia 22161 }\end{array}$ & $\begin{array}{l}\text { 20. Security Class (This } \\
\text { Page } \\
\text { UNCLASSIFIED }\end{array}$ \\
\hline FORM NTIS-35 (REV. 10-73) ENDORSED BY ANSI AND UNESCO. & THIS FORM MAY BE REPRODUCED \\
\hline
\end{tabular}




\section{TECHNIQUES FOR ESTIMATING MAGNITUDE AND FREQUENCY OF FLOODS IN MINNESOTA}

By Lowell C. Guetzkow

\section{U. S. GEOLOGICAL SURVEY}

Water-Resources Investigations 77-31

Prepared in cooperation with the

Minnesota Department of Transportation, Division of Highways and

Minnesota Department of Natural Resources, Division of Waters

May 1977 


\section{UNITED STATES DEPARTMENT OF THE INTERIOR \\ CECIL D. ANDRUS, Secretary \\ GEOLOGICAL SURVEY \\ V. E. McKelvey, Director}

For additional information write to:

U.S. Geological Survey

1033 Post Office Building

St. Paul, Minnesota 55101 
Abstract . . . . . . . . . . . . . . . . . . . 1

Introduction . . . . . . . . . . . . . . . . 2

Purpose and scope............... . . 2

Previous reports . . . . . . . . . . . . . . . 3

Gaging-station numbering system . . . . . . 3

Cooperation ................ 3

Use of metric units. . . . . . . . . . . . . . 3

Estimating flood frequency . . . . . . . . . . . . . 4

Transfer of defined flood characteristics. . . 4

Regional analysis for ungaged sites...... 13

Illustrative examples... . . . . . . . . 18

Main-stem streams . . . . . . . . . . . . 20

Accuracy and limitations . . . . . . . . . 24

Analytical techniques . . . . . . . . . . . 27

Data used . . . . . . . . . . . . . 27

Flow-frequency analysis at gaging stations . . 27

Multiple-regression model .. . . . . . . . 28

Basin characteristics investigated . . . . . 31

Summary . . . . . . . . . . . . . . . . . 31

References . . . . . . . . . . . . . . . . 33

\section{ILLUSTRATIONS}

Figure 1. Map of Minnesota showing location of gaging stations and hydrologic regions used in regression analysis.......

Figure 2. Relation of flood magnitudes for selected recurrence intervals to drainage area, Minnesota River main stem . . . . . .

Figure 3. Relation of flood magnitudes for selected recurrence intervals to drainage area, Mississippi River main stem . . . . . . 22

Figure 4. Relation of flood magnitudes for selected recurrence intervals to drainage area, Red River of the North main stem ... . 23

Figure 5. Graphical interpretation of standard error of estimate for 10-year flood in Region G............... . 25

Figure 6. Flood frequency curve for East Branch Blue Earth River near Bricelyn, Minnesota 


\section{TABLES}

$\underline{\text { Page }}$

Table 1. Conversion factors . . . . . . . . . . . 4

Table 2. Flood-frequency and basin characteristics for gaging stations in Minnesota. . . . . 5

Table 3. Regional flood-frequency equations . . . 14

Table 4. Standard error of estimates for defined relations . . . . . . . . 26 
TECHNIQUES FOR ESTIMATING MAGNITUDE

AND FREQUENCY OF FLOODS IN MINNESOTA

By Lowe11 C. Guetzkow

ABSTRACT

The magnitude and frequency of floods up to the 100-year recurrence interval can be determined for most streams in Minnesota by methods presented in this report. By multiple regression analysis, equations have been developed for estimating flood-frequency relations at ungaged sites on all natural flow streams which are not significantly affected by man-made regulation, diversion, or urbanization. Eight distinct hydrologic regions are delineated within the State with boundaries defined generally by river basin divides. In a few instances the regional divides were based on topographic or geologic considerations. Regression equations are provided for each region which relate the 2-, 5-, 10-, 25-, 50- and 100-year floods to significant basin parameters. In four regions, drainage area, slope, and storage are used as estimating variables; in two regions, drainage area and slope are used; and, in the remaining two regions, only the drainage area is used as the significant variable. Accuracy of resulting frequency estimates and limitations on the use of the equations are discussed.

For main-stem streams, which traverse regional divides and which may be affected by regulation, graphs are presented showing floods for selected recurrence intervals plotted against contributing drainage area. Flow-frequency estimates for intervening sites along the Minnesota River, Mississippi River, and the Red River of the North can be derived from these graphs.

Flood-frequency characteristics are tabulated for 201 gaging stations having 10 or more years of record. These frequency data may provide the best estimates of floods for the specified streams at sites in the vicinity of the gaging station. 


\section{INTRODUCTION}

A reliable estimate of the magnitude and frequency of floods is essential to the efficient design of bridges, culverts, dams, and other hydraulic structures. In more recent years, the need for flow-frequency estimates has greatly expanded through implementation of the State Flood Plain Management program and the Federal Flood Insurance Act.

\section{Purpose and Scope}

The purpose of this report is to provide engineers and designers with improved techniques for estimating flow-frequency relations for most streams in Minnesota. Regression equations are presented for estimating the magnitude of floods having recurrence intervals ranging from 2 to 100 years at ungaged sites on streams which are not significantly affected by man-made regulation, diversion, or urbanization. The equations apply to natural flow streams of all sizes with the exception of the main stems of the Minnesota River, Mississippi River and Red River of the North. Input to the equations requires only the measurement of selected basin characteristics which can be obtained from topographic maps of the basin under consideration.

Individual graphs are presented for the main-stem streams noted above from which selected frequency floods can be determined at ungaged sites on the basis of contributing drainage area. The effects of regulation were included in these analyses where applicable and no further adjustment is required if the degree of regulation remains unchanged.

Flood-frequency data for 201 gaged sites on natural flow streams are tabulated for use in defining flood-frequency characteristics at upstream or downstream locations. These data may be transferred by drainage area ratio and can provide an alternative to computation of flood frequency by regression equation.

Recurrence interval is the average interval of time, in years, within which the given flood magnitude can be expected to be exceeded once. It is the inverse of probability; thus a flood having an exceedance probability of 5 -percent would have a 20-year recurrence interval, and a flood having an exceedance probability of 1-percent would have a 100 -year recurrence interval.

Flow-frequency estimating methods for Minnesota presented in this report supersede those in earlier publications of the U.S. Geological Survey and the Minnesota Department of Conservation. 


\section{Previous Reports}

Previous reports by Prior (1949), Prior and Hess (1961), Wiitala (1965), and Patterson and Gamble (1968), also provided flood-frequency estimating techniques: Considerable flood data have become available since these analyses were prepared by the accumulation of additional years of record for established gaging stations, and by expansion of the gaging network through installation of crest-stage stations on small watersheds. The latter program made possible the definition of flood characteristics over a larger range in drainage area size. The additional data base and improved analytical methods warrant greater confidence in the estimates derived from the techniques provided in this report than the estimates based on previous studies.

\section{Gaging Station Numbering System}

Each gaging station has been assigned a unique number in downstream order in accordance with the permanent numbering system adopted by the U.S. Geological Survey. Stations are numbered in a downstream direction along the main stream, and stations on tributaries between main-stream stations are numbered in the order they enter the main stream. Stations on other ranks of tributaries are treated in the same manner. The complete 8-digit station number, such as 05134200 , includes the major basin part number "05" and a 6-digit station number.

\section{Cooperation}

The frequency analyses in this report were based on data collected and published by the U.S. Geological Survey as part of cooperative programs with various State and Federal agencies. The report was prepared as part of cooperative programs with the Minnesota Department of Transportation, Division of Highways and the Minnesota Department of Natural Resources, Division of Waters. Opinions, findings, and conclusions expressed in this publication are those of the U.S. Geological Survey and not necessarily those of any cooperating agency.

\section{Use of Metric Units}

The analyses and data compilations in this report are based on English units of measurement. Equivalent metric units (SI) are given in the text. Space limitations precluded the use of a dual system of units in the tables and only English units are shown. Metric units can be obtained by use of the conversion factors in table 1 . 
Table 1.--Conversion factors

The following factors may be used to convert English units published herein to the International System of units (SI).

Multiply English units

Feet $(\mathrm{ft})$

Miles (mi)

Square miles $\left(\mathrm{mi}^{2}\right)$

Cubic feet per second $\left(\mathrm{ft}^{3} / \mathrm{s}\right)$

Feet per mile $(\mathrm{ft} / \mathrm{mi})$
By .3048

1.609

2.590

.02832

.1894
To obtain SI units meters $(\mathrm{m})$

kilometers $(\mathrm{km})$

square kilometers $\left(\mathrm{km}^{2}\right)$

cubic meters per second $\left(\mathrm{m}^{3} / \mathrm{s}\right)$

meters per kilometer $(\mathrm{m} / \mathrm{km})$

\section{ESTIMATING FLOOD FREQUENCY}

It is generally accepted that the most reliable estimates of flood characteristics are those based on a frequency analysis of recorded floods at the site under consideration. Usually such records are not available and estimates must be obtained by transfer of flow-frequency data from gaged sites to the site being investigated, or must be computed from generalized floodfrequency relations.

\section{Transfer of Defined Flood Characteristics}

The flood characteristics defined by frequency analyses of gaging-station records listed in table 2 may provide the basis for satisfactory estimates at ungaged locations near the station, particularly where long-term records are available. Location of gaging stations for which frequency relations are presented are shown in figure 1. Where the period of record is short, flow-frequency estimates based on regional relations would likely provide more reliable results. Transfer of defined flow-frequency data to upstream or downstream sites on the same stream should be accomplished by an adjustment factor derivéd from drainage area ratio. Frequency data can be transferred 


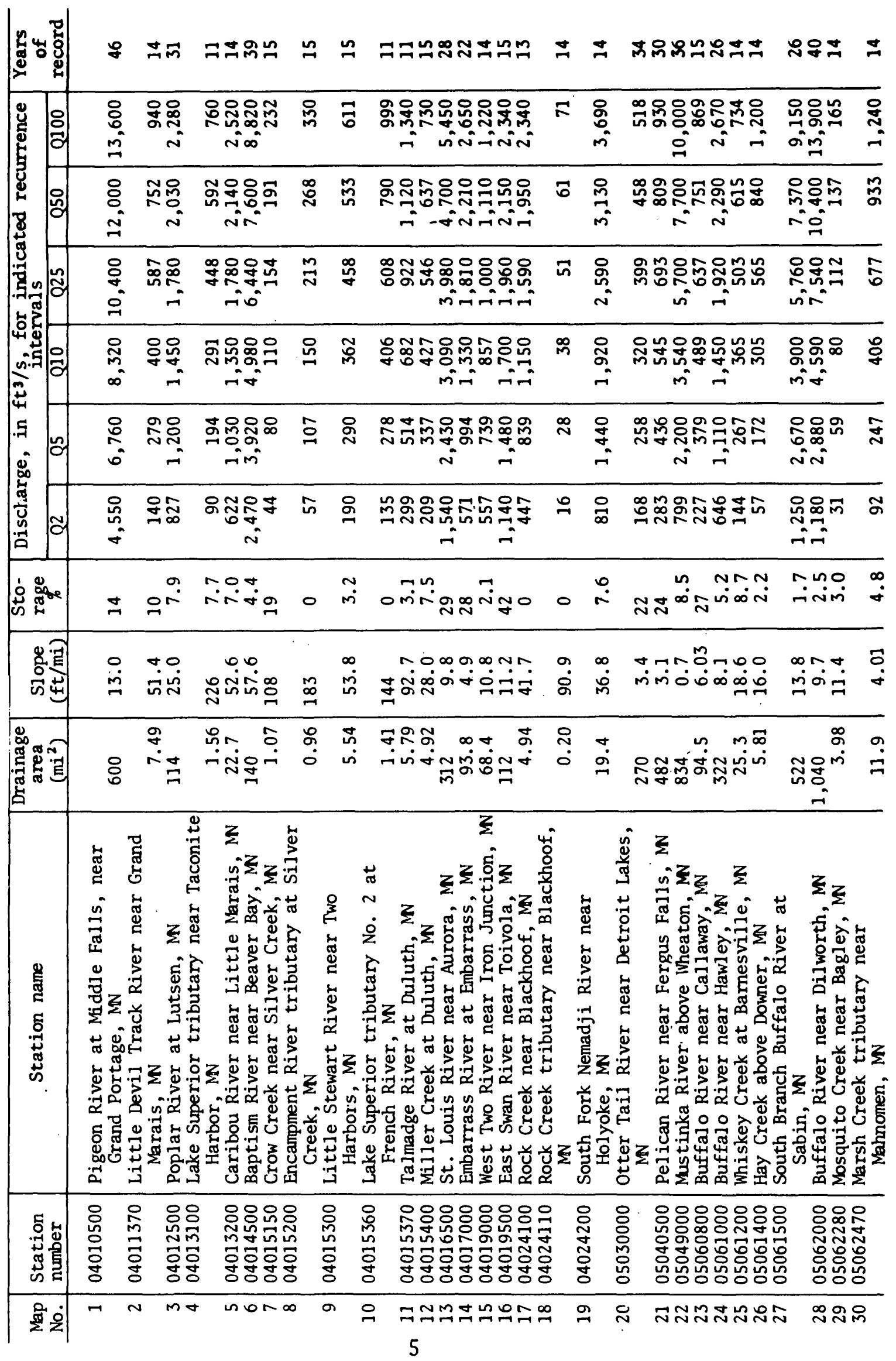




\begin{tabular}{|c|c|c|c|c|c|c|c|c|c|c|c|c|c|}
\hline 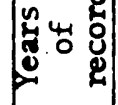 & $q$ & $\nexists m$ & $\simeq$ & 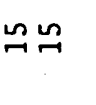 & $\stackrel{n}{n}$ & ำ & 믐유묘묘 & $\stackrel{n}{\sim}$ & $\simeq$ & $\exists \tilde{0}$ & $m=$ & 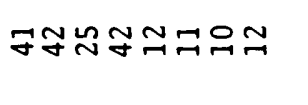 & \pm \\
\hline \multirow{6}{*}{ 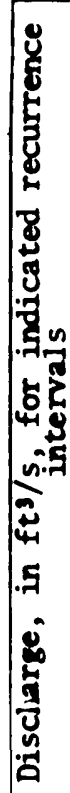 } & 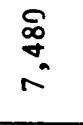 & 并贴 & $\stackrel{n}{\square}$ & సูำ & $\sqrt{2}$ & $\frac{8}{0}$ & 守壱点 & 黑 & $\underset{\infty}{\sim}$ & $\begin{array}{l}\text { 용여 } \\
\text { min } \\
\text { minn }\end{array}$ & 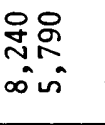 & 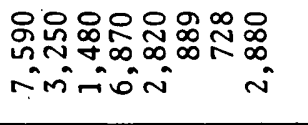 & $\Xi$ \\
\hline & $\begin{array}{l}\stackrel{\circ}{7} \\
6\end{array}$ & ño & $\bar{m}$ & 守 & $\stackrel{m}{g}$ & $\begin{array}{l}\text { 䓪 } \\
\text { n }\end{array}$ & 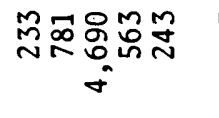 & iे & $\stackrel{0}{m}$ & 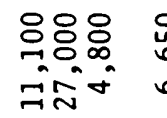 & 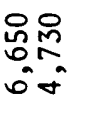 & 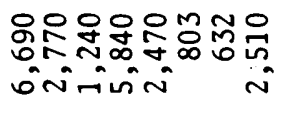 & $\stackrel{8}{q}$ \\
\hline & $\begin{array}{l}\circ \\
\infty \\
\infty \\
\text { j }\end{array}$ & 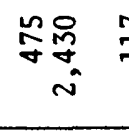 & $\bar{g}$ & 음욤 & $\approx$ & $\begin{array}{l}8 \\
8 \\
0 \\
\forall\end{array}$ & $\begin{array}{l}\text { g냄융ㅇㅇㅁ } \\
\text { m }\end{array}$ & $\stackrel{\mathbb{N}}{=}$ & กี & $\begin{array}{l}\text { 응요 } \\
\text { No요 } \\
\text { ainim }\end{array}$ & $\begin{array}{l}\text { ño } \\
\text { ñm }\end{array}$ & 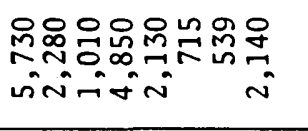 & $\underset{\sim}{\sim}$ \\
\hline & $\frac{P}{m}$ & $\stackrel{\infty}{\text { 옹 }}$ & $\stackrel{\infty}{\circ}$ & $\stackrel{\infty}{=} \stackrel{\text { 昌 }}{=}$ & $\stackrel{m}{N}$ & $\begin{array}{l}8 \\
\text { m } \\
\text { m }\end{array}$ & 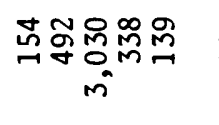 & $\stackrel{N}{\sim}$ & $\stackrel{0}{2}$ & 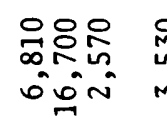 & $\begin{array}{l}\text { ํํ음 } \\
\text { nño } \\
\text { min }\end{array}$ & 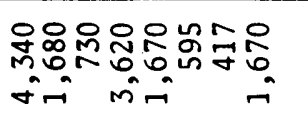 & สั \\
\hline & & 욤 & $\stackrel{m}{\infty}$ & 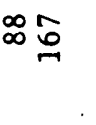 & 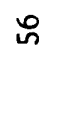 & \begin{tabular}{l} 
O⿱ \\
\multirow{2}{\sim}{} \\
$i$
\end{tabular} & 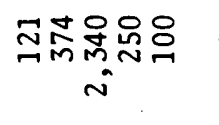 & 훔 & $\stackrel{\sim}{\sim}$ & 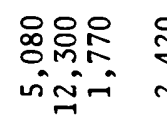 & $\begin{array}{l}\text { 守昌 } \\
\text { in } \\
\text { Nin }\end{array}$ & 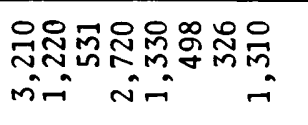 & 온 \\
\hline & $\stackrel{R}{i}$ & ำ్ำ & 용 & $g$ & $m$ & 昌 & 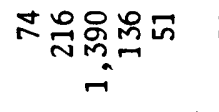 & 穴 & $\tilde{\sigma}$ & 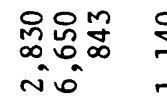 & $\begin{array}{l}\text { 웅 } \\
\text { í }\end{array}$ & 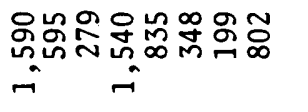 & \& \\
\hline 它 & $\stackrel{\circ}{r}$ & $\stackrel{0}{m} \dot{m}$ & $\vec{\sim}$ & 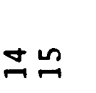 & 5 & $\bar{m}$ & O & $\begin{array}{l}\infty \\
\infty\end{array}$ & $\dddot{r}$ & $\stackrel{m}{\sim} \tilde{n}$ & $m \sim \underset{\infty}{m}$ & " & in \\
\hline$\frac{8}{8}$ & $\stackrel{\sim}{r}$ & 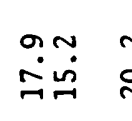 & ்ֻ & ๙̃m & $\ddot{0}$ & $\stackrel{\circ}{i}$ & RO+No & $\dot{D}$ & $\vec{m}$ & $\begin{array}{l}\text { ONun } \\
\text { \&Nin }\end{array}$ & vis & moon் & $\stackrel{?}{\infty}$ \\
\hline हैं & $\begin{array}{l}\infty \\
\infty \\
\infty\end{array}$ & 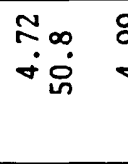 & $\begin{array}{l}\text { gे } \\
\dot{\sigma}\end{array}$ & $\begin{array}{l}\text { \& } \\
\text { iñ }\end{array}$ & $\stackrel{\oplus}{\check{r}}$ & ๙ૂ & 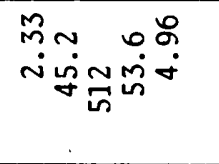 & $\begin{array}{l}\tilde{o} \\
\dot{0}\end{array}$ & $\tilde{n}$ & 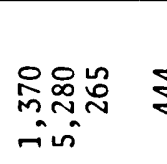 & \& & 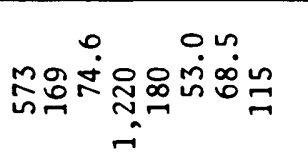 & $\stackrel{m}{\sigma}$ \\
\hline 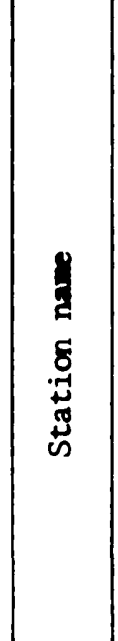 & 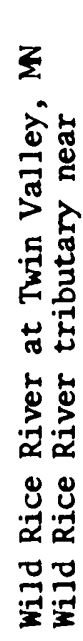 & 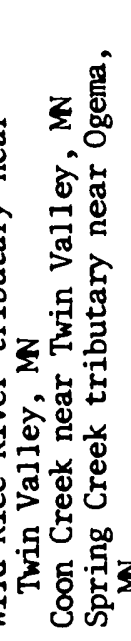 & 莙 & 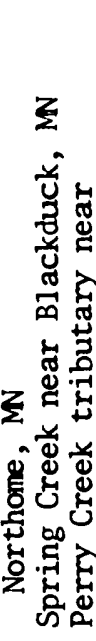 & 告 & $\begin{array}{c}0 \\
0 \\
0 \\
0\end{array}$ & 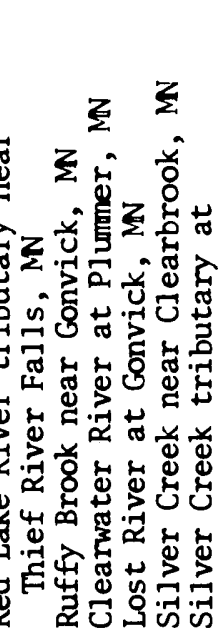 & 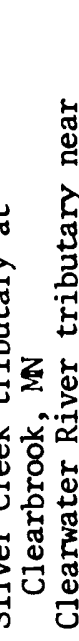 & 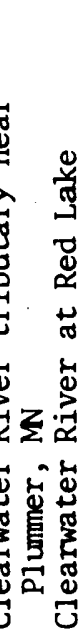 & 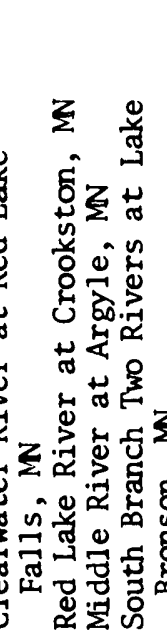 & 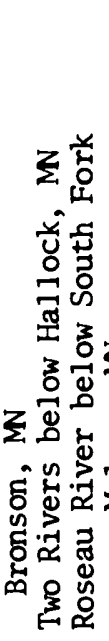 & 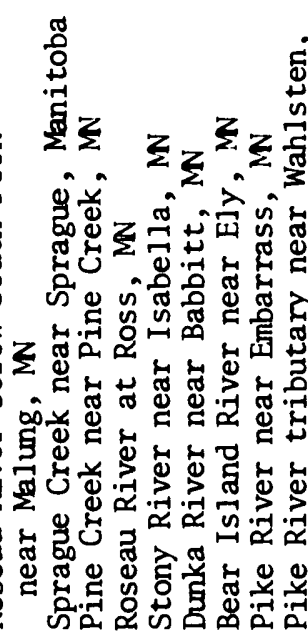 & 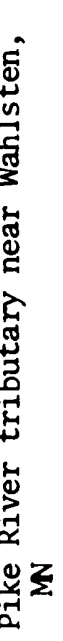 \\
\hline 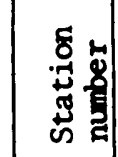 & 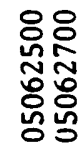 & 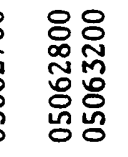 & 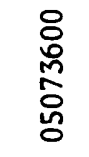 & 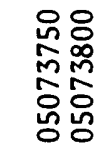 & $\begin{array}{l}8 \\
8 \\
0 \\
0 \\
\text { ํํㅇ }\end{array}$ & $\begin{array}{l}\qquad \\
c \\
c \\
c \\
\\
\mathfrak{c} \\
c\end{array}$ & 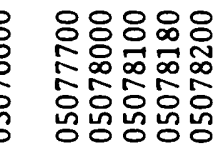 & $\begin{array}{l}8 \\
\text { + } \\
0 \\
\text { I } \\
\text { 이 }\end{array}$ & 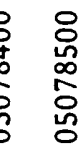 & 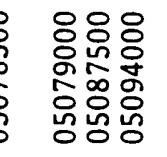 & 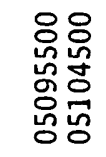 & 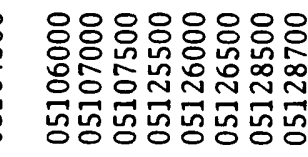 & \\
\hline 要定 & $\bar{m} \tilde{m}$ & m留 & $\stackrel{m}{m}$ & 号向 & mi & ஜ & 乎尔孚 & t & $f$ & $\tilde{\sigma}+\infty$ & 웃도 & ing & \\
\hline
\end{tabular}




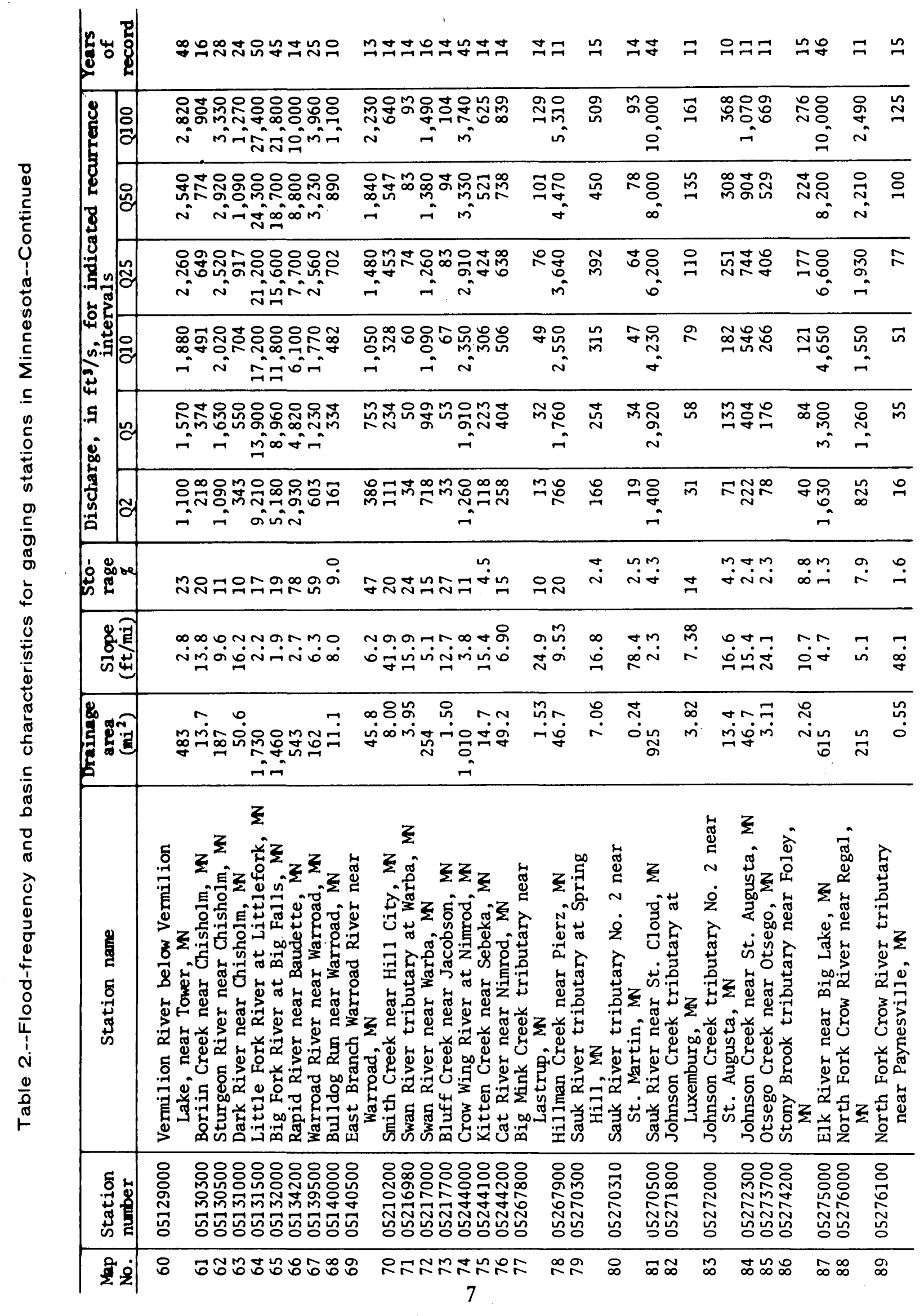




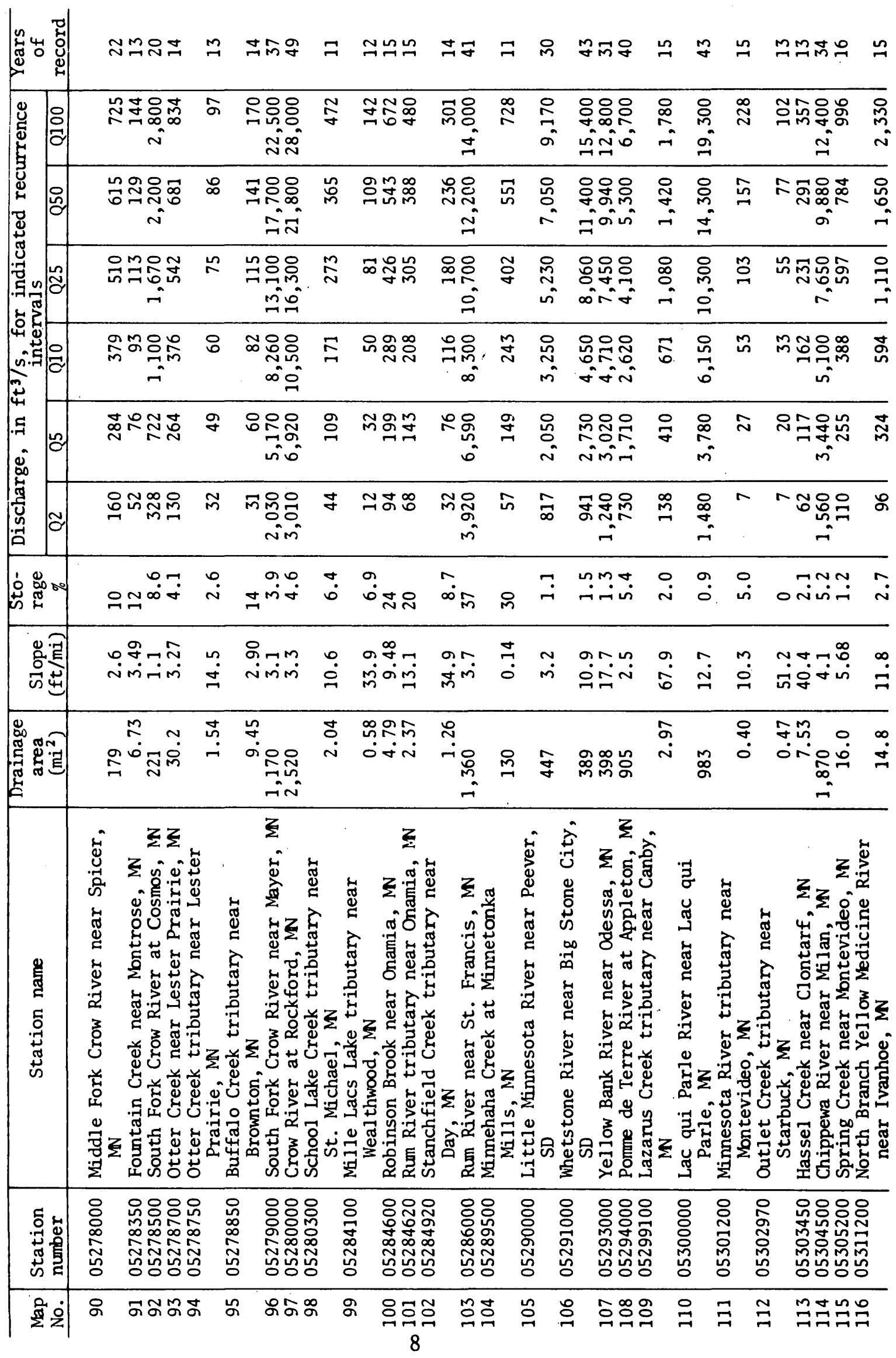




\begin{tabular}{|c|c|c|c|c|c|c|c|c|c|c|c|c|c|c|}
\hline 苟出 & $\stackrel{\sim}{7}$ & $\stackrel{n}{\sim}$ & ले & 兄品品尔 & $\stackrel{\circ}{\circ}$ & $\stackrel{0}{\longrightarrow}$ & エニ出す & 요 & $\stackrel{\sim}{\sim} \cong \mathbb{N}$ & $\stackrel{0}{\sim}$ & $\stackrel{\circ}{\sim}$ & 兄さ & $\Xi$ & $\stackrel{2}{2} \underset{\sim}{n}$ \\
\hline \multirow{6}{*}{ 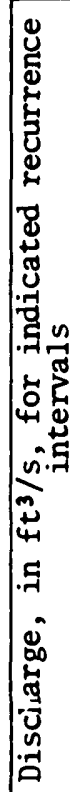 } & $\underset{7}{\rightleftarrows}$ & $\stackrel{i}{m}$ & $\begin{array}{l}8 \\
\vdots \\
i \\
\text { i }\end{array}$ & 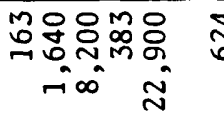 & तु욤 & $\stackrel{\stackrel{N}{N}}{\sim}$ & 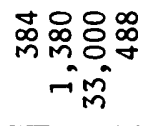 & $\begin{array}{l}0 \\
\infty \\
\sim \\
\sim\end{array}$ & 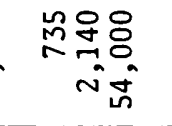 & 욤 & $\underset{N}{N}$ & 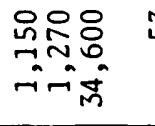 & $\stackrel{m}{n}$ & mo \\
\hline & $\stackrel{g}{\Rightarrow}$ & $\underset{\sim}{\mathbb{\infty}}$ & $\begin{array}{l}8 \\
8 \\
\circ \\
0\end{array}$ & 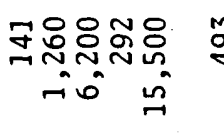 & $\begin{array}{r}\text { mo } \\
\text { go } \\
\text { - }\end{array}$ & $\stackrel{\substack{\infty \\
\sim}}{\sim}$ & $\begin{array}{c}\text { 꽁ㅇㅇㅇ } \\
\text { N엉 } \\
\text { riN }\end{array}$ & $\stackrel{?}{\stackrel{2}{\sim}}$ & $\begin{array}{c}\text { 구웅ㅇㅇ } \\
\text { rif }\end{array}$ & 오 & $\frac{5}{\sigma}$ & 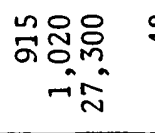 & $\stackrel{\infty}{+}$ & 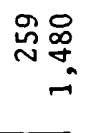 \\
\hline & $\stackrel{\infty}{\circ}$ & $\stackrel{M}{N}$ & $\begin{array}{l}\stackrel{8}{1} \\
\approx \\
\approx\end{array}$ & 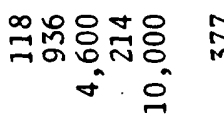 & în & 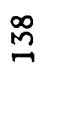 & 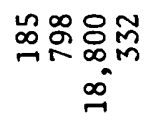 & $\frac{7}{7}$ & $\begin{array}{l}\text { 웅융 } \\
\text { riñ }\end{array}$ & $\stackrel{m}{r}$ & 옥 & $\begin{array}{r}\text { 동ㅇㅇㅇ } \\
\text { ᄋ }\end{array}$ & $\stackrel{m}{\forall}$ & 유유 \\
\hline & 요 & $\stackrel{\infty}{\infty}$ & $\begin{array}{l}8 \\
8 \\
+ \\
0\end{array}$ & 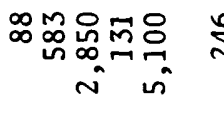 & 过品 & $\infty$ & 음 & g & 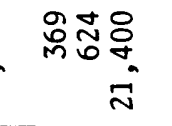 & m & 喕 & mon̊ & m & $\underset{\sim}{\forall} \underset{\sim}{\infty}$ \\
\hline & $g$ & $\stackrel{\infty}{\sim}$ & $\begin{array}{l}\text { 옹 } \\
\text { m }\end{array}$ & 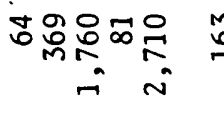 & m. & กี & 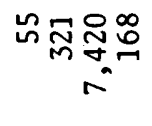 & $\stackrel{n}{n}$ & 중요 & $\stackrel{\infty}{m}$ & $\stackrel{\sim}{\infty}$ & 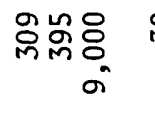 & mి & 욤 \\
\hline & $\approx$ & $\bar{\sigma}$ & $\begin{array}{l}\circ \\
\stackrel{0}{\because} \\
-1\end{array}$ & 阽守的云 & $\Gamma \stackrel{\infty}{\infty}$ & $N$ & $\begin{array}{c}\text { 픔용 } \\
\text { N }\end{array}$ & $\stackrel{N}{m}$ & 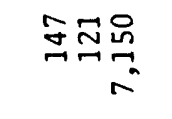 & 요 & 5 & î̀ㅁㅇㅛ & $\vec{N}$ & 요쑈 \\
\hline 造离用 & 0 & $\ddot{0}$ & $\ddot{0}$ & 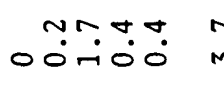 & $\ddot{m}$ & 0 & $00 \hat{0}$ & & 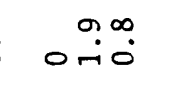 & 0 & $\stackrel{0}{\dot{m}}$ & 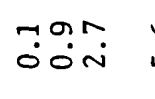 & $\stackrel{\varphi}{\dot{0}}$ & $\cong \Xi$ \\
\hline 范 & $\stackrel{\vdots}{\infty}$ & $\dot{m}$ & $\stackrel{+}{\sim}$ & 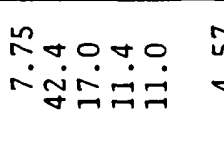 & 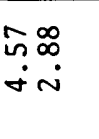 & $\begin{array}{l}\infty \\
\stackrel{\sim}{\sim}\end{array}$ & 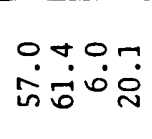 & $N$ & 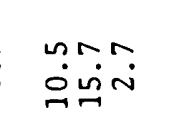 & $\stackrel{\infty}{\stackrel{\infty}{n}}$ & $\begin{array}{l}\tilde{O} \\
\dot{\sigma}\end{array}$ & 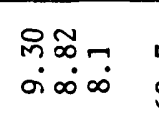 & $\ddot{\infty}$ & ㅇ. \\
\hline 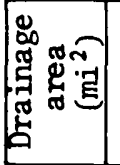 & $\stackrel{m}{m}$ & $\stackrel{?}{\dot{m}}$ & นึู & $\begin{array}{c}m \infty \\
\infty \\
0 \\
0 \\
0\end{array}$ & \begin{tabular}{l}
$\sim m$ \\
\multirow{n}{*}{}
\end{tabular} & б. & im & $\tilde{m}$ & 우요 & ó & $\stackrel{n}{i}$ & 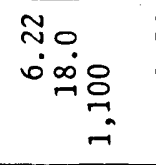 & in. & $\stackrel{\circ}{\sim} \underset{\sim}{\sim}$ \\
\hline 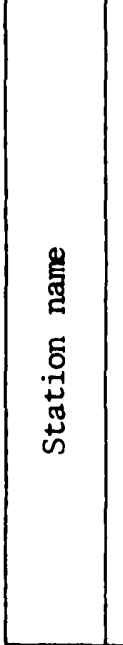 & 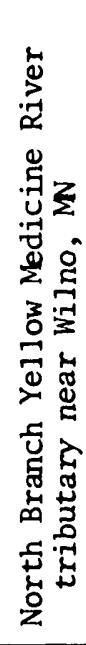 & 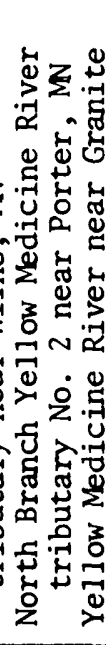 & 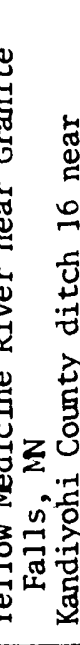 & 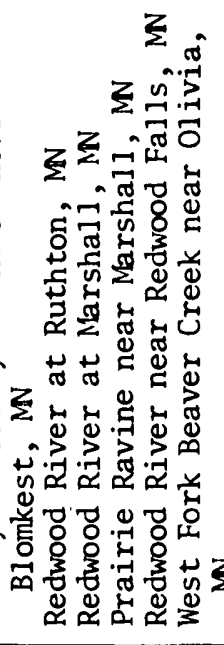 & 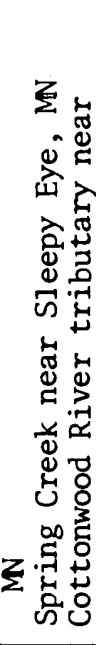 & 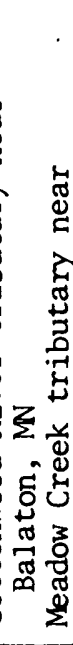 & 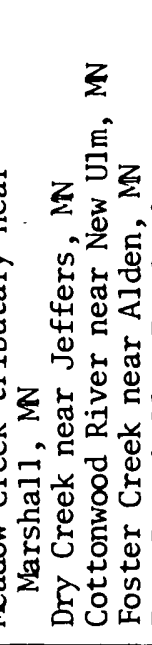 & 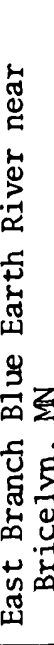 & 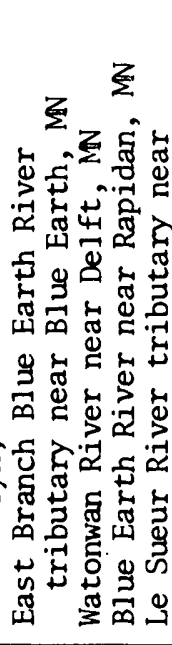 & 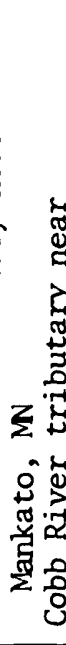 & 㝴 & 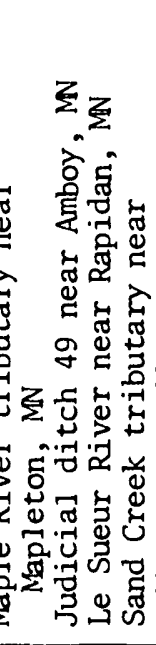 & 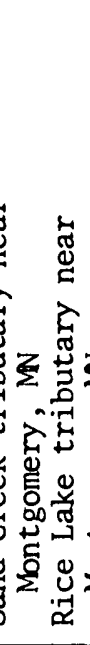 & 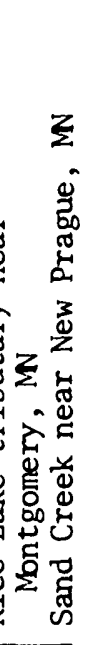 \\
\hline 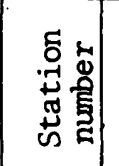 & 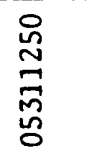 & 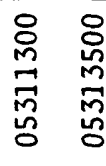 & 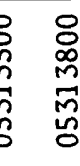 & 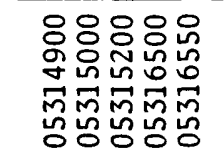 & 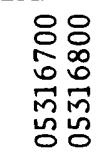 & 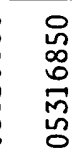 & 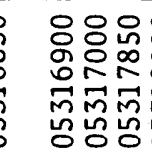 & & 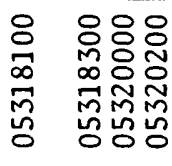 & $\begin{array}{l}\text { ○ } \\
\text { m } \\
\text { กิ } \\
\text { m̃ } \\
\text { ñ }\end{array}$ & $\begin{array}{l}\text { O } \\
\text { 寸 } \\
\text { N } \\
\text { in }\end{array}$ & 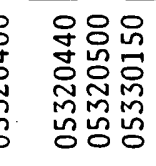 & 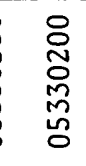 & 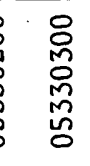 \\
\hline 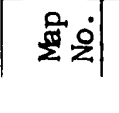 & $\cong$ & $\stackrel{\infty}{\Rightarrow} \cong$ & 욕 & 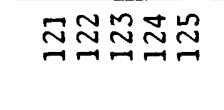 & $\underset{\sim}{\stackrel{N}{N}}$ & $\stackrel{\infty}{\sim}$ & 옴욤 & & 紡 & $\hat{m}$ & 4 & 兽昌示 & $\stackrel{\sim}{\sim}$ & $\underset{m}{M}$ \\
\hline
\end{tabular}




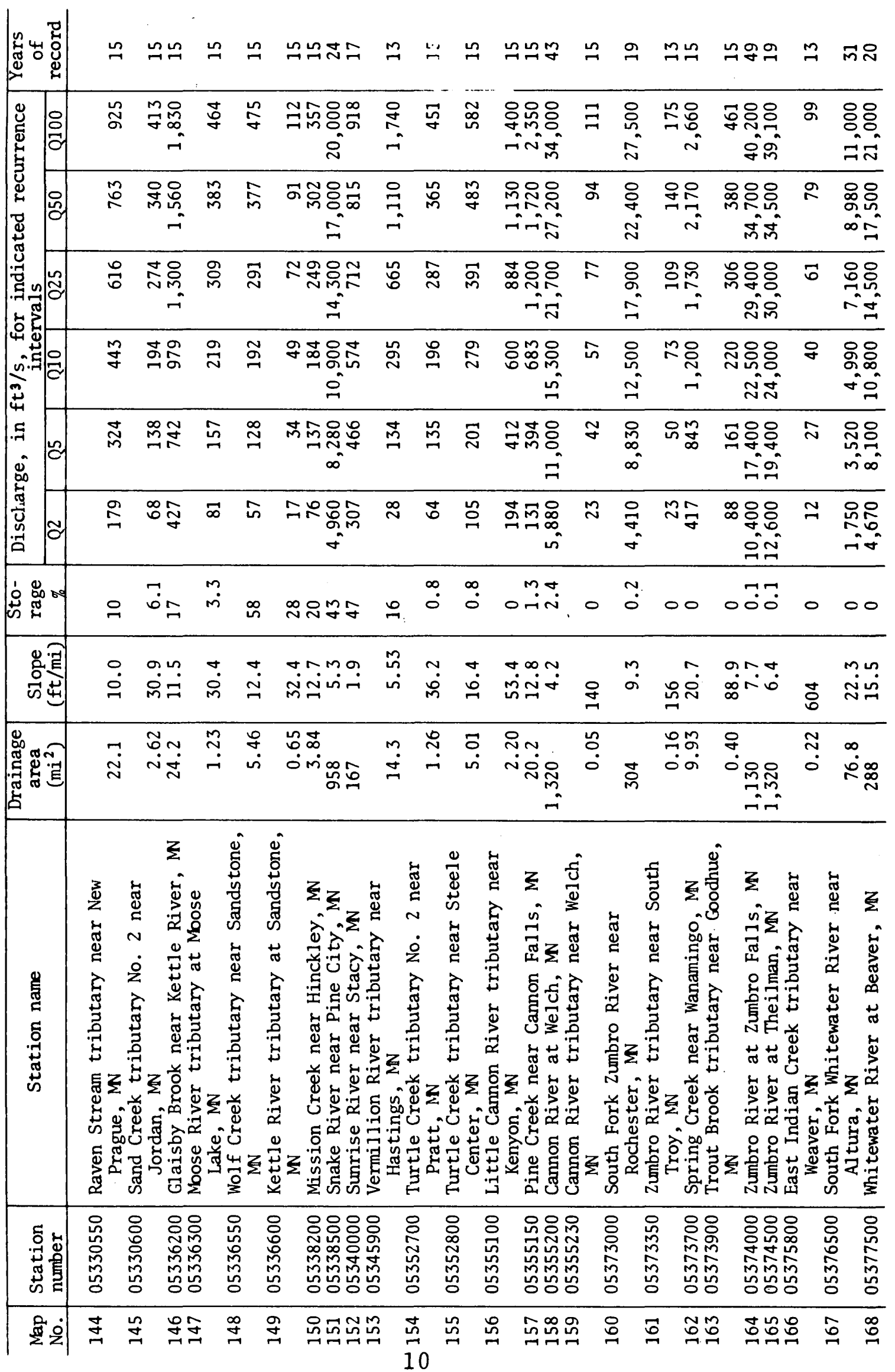




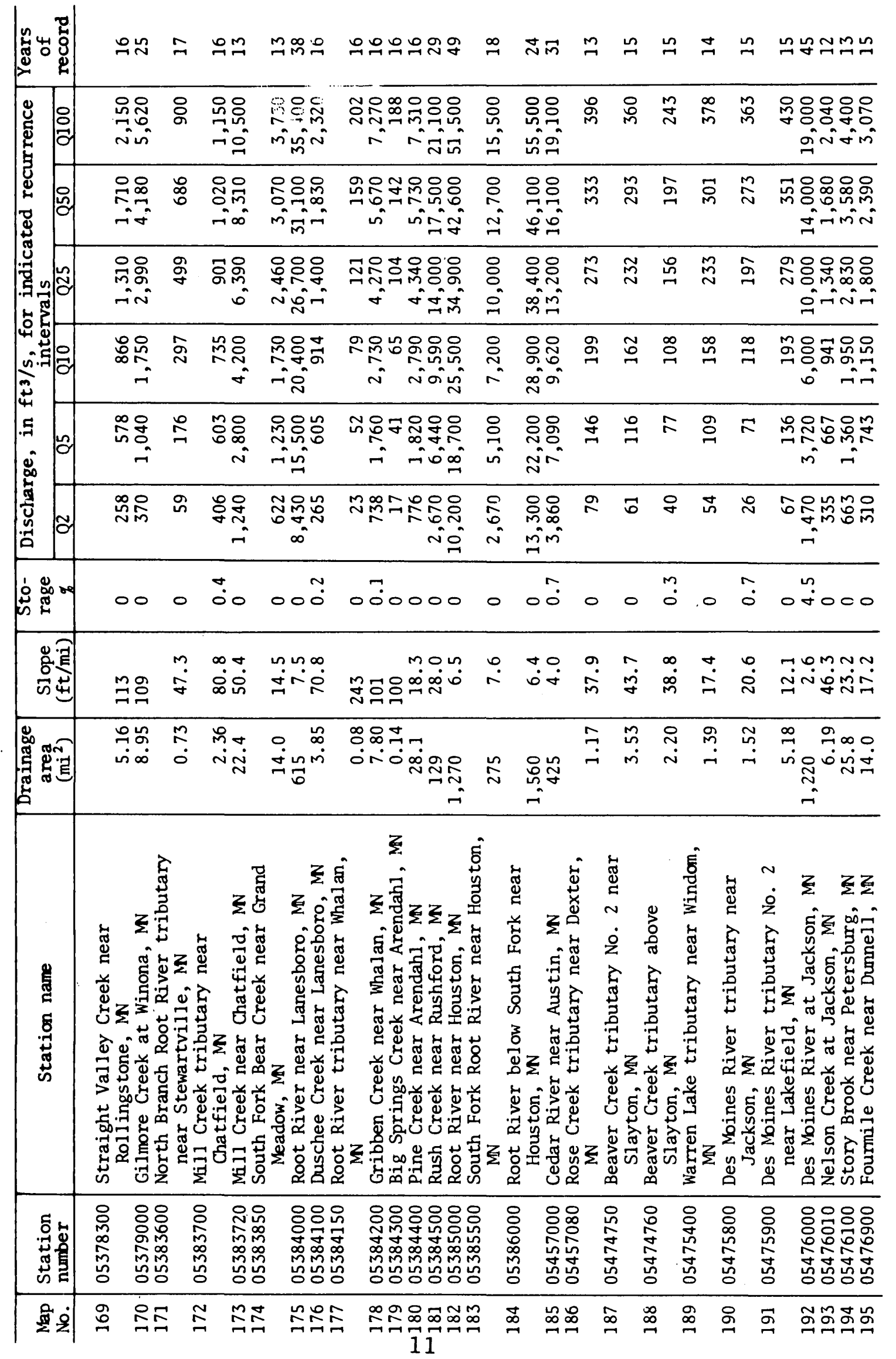




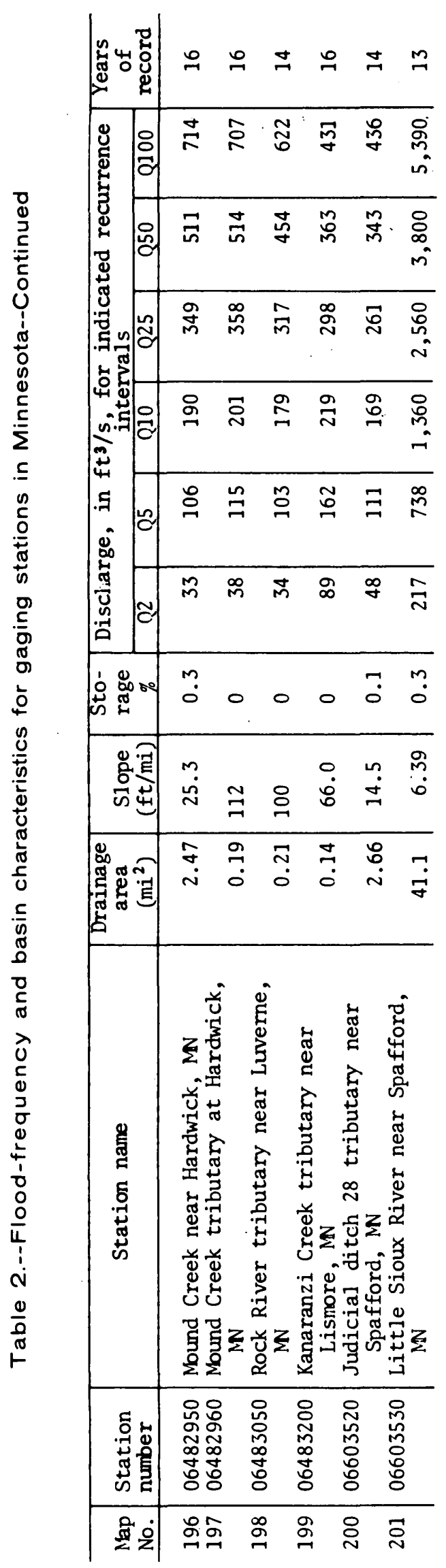


most reliably by the following relation which is applicable to most areas in Minnesota.

$$
Q_{u}=Q_{g}\left(A_{u} / A_{g}\right)^{0 \cdot 6}
$$

where: $Q_{u}$ is the flood-frequency estimate for the ungaged site

$Q_{g}$ is the flood-frequency value for the gaged site

$A_{u}$ is the drainage area for the ungaged site

$A_{g}$ is the drainage area for the gaged site

Local conditions may warrant a slight increase or decrease of the 0.6 exponent, which must be based on engineering judgment. Use of the transfer relation should be limited to sites which differ in drainage area size by no more than 40 percent from the gaged site.

\section{Regional Analyses for Ungaged Sites}

Equations derived from multiple regression analyses can be used to obtain flood-frequency estimates for ungaged sites on natural flow streams. Peak discharges for the selected recurrence intervals can be computed from these mathematical equations tabulated in table 3 , which relate flood magnitude to basin characteristics. A set of equations to estimate flood peaks for the 2-,5-, 10-, 25-, 50-, and 100-year recurrence intervals (identified as $Q_{2}, Q_{5}, Q_{10}$, etc.) are provided for each of the hydrologic regions into which the State has been divided. The eight regions are outlined on figure 1 . The regional boundaries cannot be defined precisely; therefore, where the estimating site falls close to a regional divide, consideration should be given to averaging the results obtained by computation of the flood magnitudes from the equations for the two adjoining regions. Particular care should be exercised when the site in question has a large value for a basin characteristic that is not used in the regression relation for both regions. If a flood-frequency estimate is to be made downstream from a regional divide which the stream crosses, the discharge at the site should be determined by weighting the regression estimates computed from both regional relations according to drainage area.

Due regard should be given to the limitations of these equations as discussed in a following section (Accuracy and Limitations). 


$Q_{2}=29.2 \mathrm{~A} .62$
$Q_{5}=54.2 \mathrm{~A} .62$
$Q_{10}=73.8 \mathrm{~A} .62$
$Q_{25}=101 \mathrm{A.62}$
$Q_{50}=124 \mathrm{A.62}$
$Q_{100}=149 \mathrm{A.62}$

Region B

$$
\begin{aligned}
& Q_{2}=5.71 \mathrm{~A} .660 \mathrm{~S} .407 \mathrm{St}^{-.027} \\
& Q_{5}=16.1 \mathrm{~A} .646 \mathrm{~S} .452 \mathrm{St}^{-.231} \\
& Q_{10}=26.8 \mathrm{~A} .642 \mathrm{~S} .473 \mathrm{St}^{-.333} \\
& Q_{25}=46.5 \mathrm{~A} .636 \mathrm{~S} .492 \mathrm{St}^{-.043} \\
& Q_{50}=65.2 \mathrm{A.634} \mathrm{S} .505 \mathrm{St}^{-} .513 \\
& Q_{100}=88.4 \mathrm{~A} .631 \mathrm{~S} .516 \mathrm{St}^{-.575}
\end{aligned}
$$

$\underline{\text { Region C }}$

$Q_{2}=10.5 A .764 \mathrm{~S} .375$

$Q_{5}=15.9 A .736 \mathrm{~S} .421$

$$
Q_{10}=19.8 \mathrm{~A} .722 \mathrm{~S} \cdot 447
$$$$
Q_{25}=24.5 \mathrm{~A} .708 \mathrm{~S} .476
$$$$
Q_{50}=28.1 \mathrm{~A} .699 \mathrm{~S} .495
$$$$
Q_{100}=32.0 \mathrm{~A} .690 \mathrm{~S} .512
$$

\section{Region D}

$$
\begin{aligned}
& Q_{2}=7.90 \mathrm{~A} .654 \mathrm{~S} .356 \\
& Q_{5}=25.1 \mathrm{~A} .666 \mathrm{~S}^{288} \mathrm{St}^{-} .175 \\
& Q_{10}=44.8 \mathrm{~A} .673 \mathrm{~S} .252 \mathrm{St}^{-} .265 \\
& Q_{25}=79.7 \mathrm{~A} .682 \mathrm{~S} .217 \mathrm{St}^{-} .354 \\
& Q_{50}=115 \mathrm{~A} .688 \mathrm{~S} .194 \mathrm{St}^{-} .411 \\
& Q_{100}=157 \mathrm{~A} .695 \mathrm{~S} .175 \mathrm{St}^{-} .460
\end{aligned}
$$

\section{Region E}

$Q_{2}=1.91 \mathrm{A.913} \mathrm{S} .803$

$Q_{5}=5.76 A .852 \mathrm{~S} .774$

$Q_{10}=9.83 \mathrm{A.821} \mathrm{S} .725$

$Q_{25}=17.0 \mathrm{A.790} \mathrm{S.674}$

$Q_{50}=23.9 A .770 \mathrm{~S} .644$

$Q_{100}=32.4 \mathrm{A.753} \mathrm{S} .616$

\section{Region F}

$Q_{2}=83.8 \mathrm{~A} \cdot 47$

$Q_{5}=208 A \cdot 49$

$Q_{10}=322 A .50$

$Q_{25}=487 A \cdot 51$

$Q_{50}=580 A \cdot 52$

$Q_{100}=762 A .52$

Region G

$Q_{2}=15.8 \mathrm{~A}^{687} \mathrm{~S}^{253} \mathrm{St}^{-.115}$

$Q_{5}=32.1 \mathrm{~A}^{723} \mathrm{~S}^{294} \mathrm{St}^{-.212}$

$Q_{10}=45.6 \mathrm{~A}^{.742} \mathrm{~S}^{313} \mathrm{St}^{-.250}$

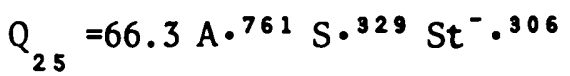

$Q_{50}=83.5 \mathrm{~A}^{.774} \mathrm{~S}^{340} \mathrm{St}^{-.} 337$

$Q_{100}=102 \mathrm{~A} \cdot 786 \mathrm{~S} \cdot 349 \mathrm{St}^{-.363}$

\section{Region $\mathrm{H}$}

$Q_{2}=23.2 \mathrm{A.787} \mathrm{S} .348^{-3 \mathrm{St}^{-} .753}$

$Q_{5}=55.0 \mathrm{~A}^{.753} \mathrm{~S}^{324} \mathrm{St}^{-} .640$

$Q_{10}=86.4 \mathrm{~A} .735 \mathrm{~S}^{309} \mathrm{St}^{-.584}$

$Q_{25}=135 \mathrm{~A} .716 \mathrm{~S} .295 \mathrm{St}^{-.520}$

$Q_{50}=183 \mathrm{~A}^{.704} \mathrm{~S}^{283} \mathrm{St}^{-.481}$

$Q_{100}=236 \mathrm{A.694} \mathrm{S}^{.274} \mathrm{St}^{-.447}$ 
Variables used for the equations in table 3 have the following measurement units:

$\mathrm{Q}_{\mathrm{T}}$ - Peak discharge for $\mathrm{T}$-year recurrence interval, in cubic feet per second.

A - Drainage area, in square miles.

S - Average main channel slope, between 10 and 85 percent points, in feet per mile.

St - Area of lakes, ponds and swamps, expressed as percentage of drainage area and increased by 1 percent.

Values for the basin characteristics used in the regression analyses were determined by the methods outlined below. Required independent variables used for estimated flood characteristics at ungaged sites should be determined in a like manner.

1. Drainage area.- Trace contributing drainage-area outline on topographic maps along divides indicated by contour elevations, starting at point on stream where frequency characteristics are to be defined.

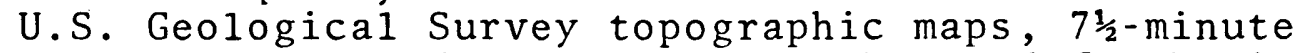
or 15-minute quadrangles, should be used for basins smaller than 150 square miles. For larger basins, the $1: 250,000$ scale topographic maps may be used. Planimeter the outlined area to obtain drainage area (A) in square miles.

2. Main channel slope.- Determine the main channel of the stream on the drainage area map, from the point selected for the frequency estimate, to the extreme rim of the basin. Extension of the main channel to the basin divide, beyond the upstream end of the defined stream, should be made as indicated by contours. Upstream from each stream junction, choose the main channel as the fork which drains the larger area. Measure the total length by dividers set at appropriate intervals, such as $0.1 \mathrm{mile}$ for $7 \frac{1}{2}$-minute quadrangles, 0.25 mile for 15 -minute quadrangles, and 0.5 mile for the $1: 250,000 \mathrm{scale}$ maps. Locate points 10 and 85 percent of the main channel length upstream from the point of interest, and determine the elevation of these points by interpolation between contours. The average main channel slope $(S)$ is computed as the difference in elevation, in feet, divided by the length, in miles, between the 10 and 85 percent points. 


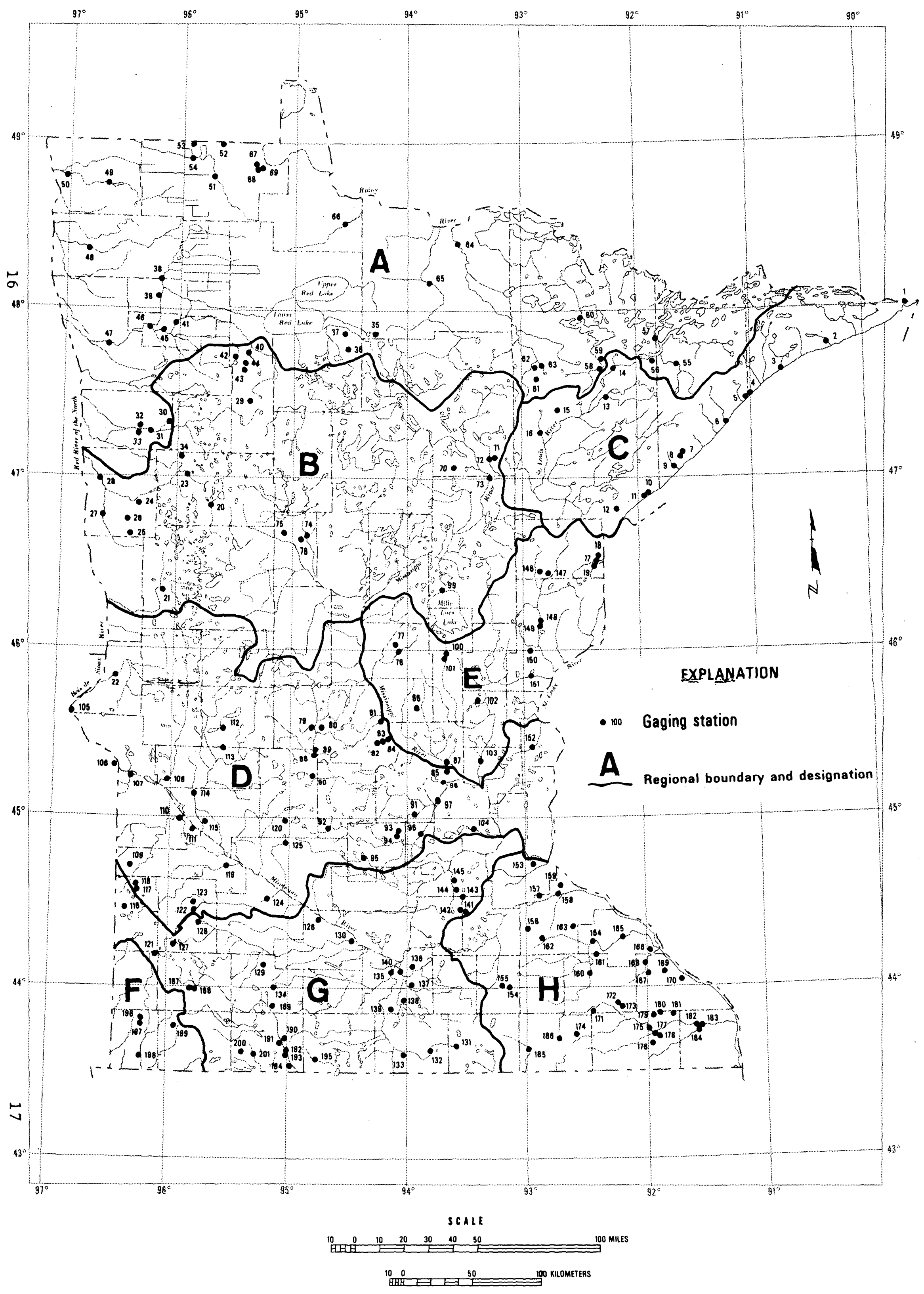

Figure 1.--Map of Minnesota showing location of gaging stations and hydrologic regions used in regression analysis. 
3. Storage.- Measure the area of lakes, ponds and swamps in the drainage basin on topographic maps. Storage areas can be measured by planimeter or by using a transparent grid. The grid is placed over the water and swamp areas and the number of squares, and estimated fractional parts of squares, are summed up and multiplied by the area of each square, as calculated for the scale of the map being used. The total area of lakes, ponds and swamps is expressed as a percentage of the total contributing area. This percentage is then increased by 1 percent to obtain the storage parameter (St) used in the equations.

A transparent grid suitable to most map scales is enclosed in a packet at the back of this report.

\section{Illustrative Examples}

The following examples illustrate use of the relations to compute flow frequency estimates.

Example 1.- Estimate the 50-year flood on the Sauk River at Cold Spring, an ungaged site.

Solution:

1) Inspection of figure 1 and table 2 indicate the availability of gaging-station data for the Sauk River in close proximity to Cold Spring. The station is identified as Sauk River near St. Cloud, map no. 81 (Station No. 05270500).

2) Contributing drainage area at Cold Spring is planimetered on topographic maps as $832 \mathrm{mi}^{2}$.

3) Reduction in drainage area at Cold Spring is only 10 percent from the $925 \mathrm{mi}^{2}$ listed in table 2 for the St. Cloud gaging station. Therefore, a transfer of flood characteristics by drainage area ratio is appropriate.

4) From table 2, $Q_{g}=8,000 \mathrm{ft}^{3} / \mathrm{s}$ for 50-year flood.

5) By substitution into transfer equation:

$$
\begin{aligned}
Q_{u}=Q_{g}\left(A_{u} / A_{g}\right)^{0.6} \\
Q_{50}=8,000(832 / 925)^{0.6} \\
Q_{50}=8,000 \times 0.938 \\
Q_{50}=7,500 \mathrm{ft}^{3} / \mathrm{s}\left(212 \mathrm{~m}^{3} / \mathrm{s}\right)
\end{aligned}
$$


Example 2.- Estimate the 25-year peak discharge for an ungaged site on Spring Creek in Swift County, at the crossing of State Highway $9,3 \frac{1}{2}$ miles west of Sunburg.

1) Inspection of figure 1 and table 2 indicate that no gaging-station data are available on this stream, therefore, flow-frequency estimates must be derived from regional equations.

2) Site is identified from figure 1 as being in Region D. Applicable equation for 25 -year flood is located in table 3 .

3) Drainage area is outlined on topographic map, De Graff SE $7 \frac{1}{2}-$ minute quadrangle.

4) Drainage area (A) is planimetered as $1.28 \mathrm{mi}^{2}$, and main channel length is measured as $1.49 \mathrm{mi}$ to the watershed divide.

5) The main channel slope is computed by dividing the difference in elevations at mile $0.15(0.10 \times 1.49)$ and mile $1.27(0.85 \times 1.49)$ by $1.12(1.27-0.15)$, the distance between the two points.

Elevation at mile 1.27 is $1235 \mathrm{ft}$

Elevation at mile 0.15 is $1212 \mathrm{ft}$

Ma in channel slope $(S)=(1235-1212) / 1.12=20.5$ $\mathrm{ft} / \mathrm{mi}$

6) Total lake, pond and swamp area is determined from the map by the grid system described in the discussion on storage preceding example 1 . Fifteen of the small grid squares are counted as storage area.

15 squares $\times 0.00144 \mathrm{mi}^{2}=0.02 \mathrm{mi}^{2}$

$$
\text { Storage }=\frac{0.02}{1.28} \times 100=1.6 \text { percent }
$$

Storage index $(S t)=1.6+1.0=2.6$ percent

7) Region $D$ equation for 25-year flood from table 3:

$$
Q_{25}=79.7 \mathrm{~A}^{682} \mathrm{~S}^{217} \mathrm{St}^{-} .354
$$

8) By substitution of the variables:

$$
Q_{25}=79.7(1.28) .682(20.5) .217(2.6)^{-.354}
$$




$$
Q_{25}=130 \mathrm{ft}^{3} / \mathrm{s}\left(3.68 \mathrm{~m}^{3} / \mathrm{s}\right)
$$

Plotting points to define a frequency curve for the site can be obtained by solution of equations for other recurrence intervals.

\section{Main-stem Streams}

Estimating relations given previously do not apply to the main stem of the Minnesota River, Mississippi River and Red River of the North. The effects of regulation, interregional character of the streams and (or) the large drainage areas involved, require unique definition of the flood characteristics for these streams. Individual relations between flood magnitude and contributing drainage area were prepared based on interpolation between gage sites on the main stems. Floodfrequencies indicated for regulated reaches of the main-stem streams are based on the assumption that past records represent homogeneous regulation patterns and are applicable only if such regulation patterns remain unchanged in the future.

The 100-year flood estimates for the Minnesota River, Red River of the North, and Mississippi River (between Aitkin and St. Paul) have been coordinated under an interagency agreement. Agencies involved in this coordination process, with limitations imposed by their area of interest or jurisdiction, are as follows: St. Paul District-Corps of Engineers, Soil Conservation Service, U.S. Geological Survey, Minnesota Department of Natural Resources, and North Dakota Water Commission (Red River of the North Regional Flood Analysis, 1971).

Graphs showing flood estimates for selected recurrence intervals versus contributing drainage area for the Minnesota River, Mississippi River, and Red River of the North are presented in figures $2-4$, respectively. When using the frequency graph for the Red River, it should be recognized that plotting positions of drainage areas from Halstad to Emerson have been corrected by subtracting $3,800 \mathrm{mi}^{2}\left(9842 \mathrm{~km}^{2}\right)$ for closed basins in the Sheyenne River basin in North Dakota.

Definition of flood characteristics for the main stem of the St. Croix River from St. Croix Falls to Prescott, Wisconsin is very complex owing to regulation of flows and the backwater effect from the Mississippi River, which affects elevationfrequency relations through a large part of this reach. Such analyses are outside the scope of this report. Frequency data for this section of the St. Croix River can be obtained from the report "St. Croix River Regional Flood Analysis" (Wiitala, 1973 ). 


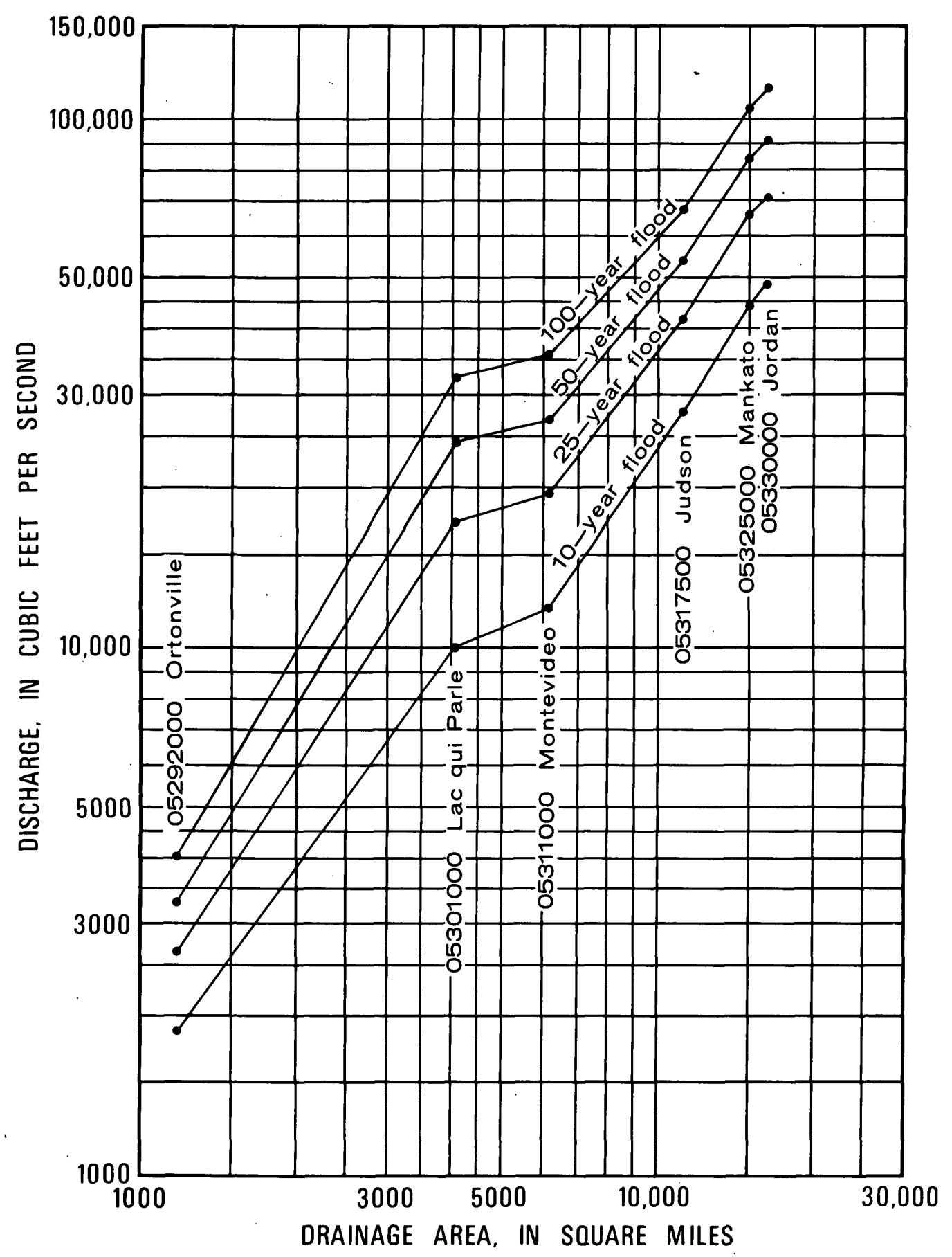

Figure 2.--Relation of flood magnitudes for selected recurrence intervals to drainage area, Minnesota River main stem. 


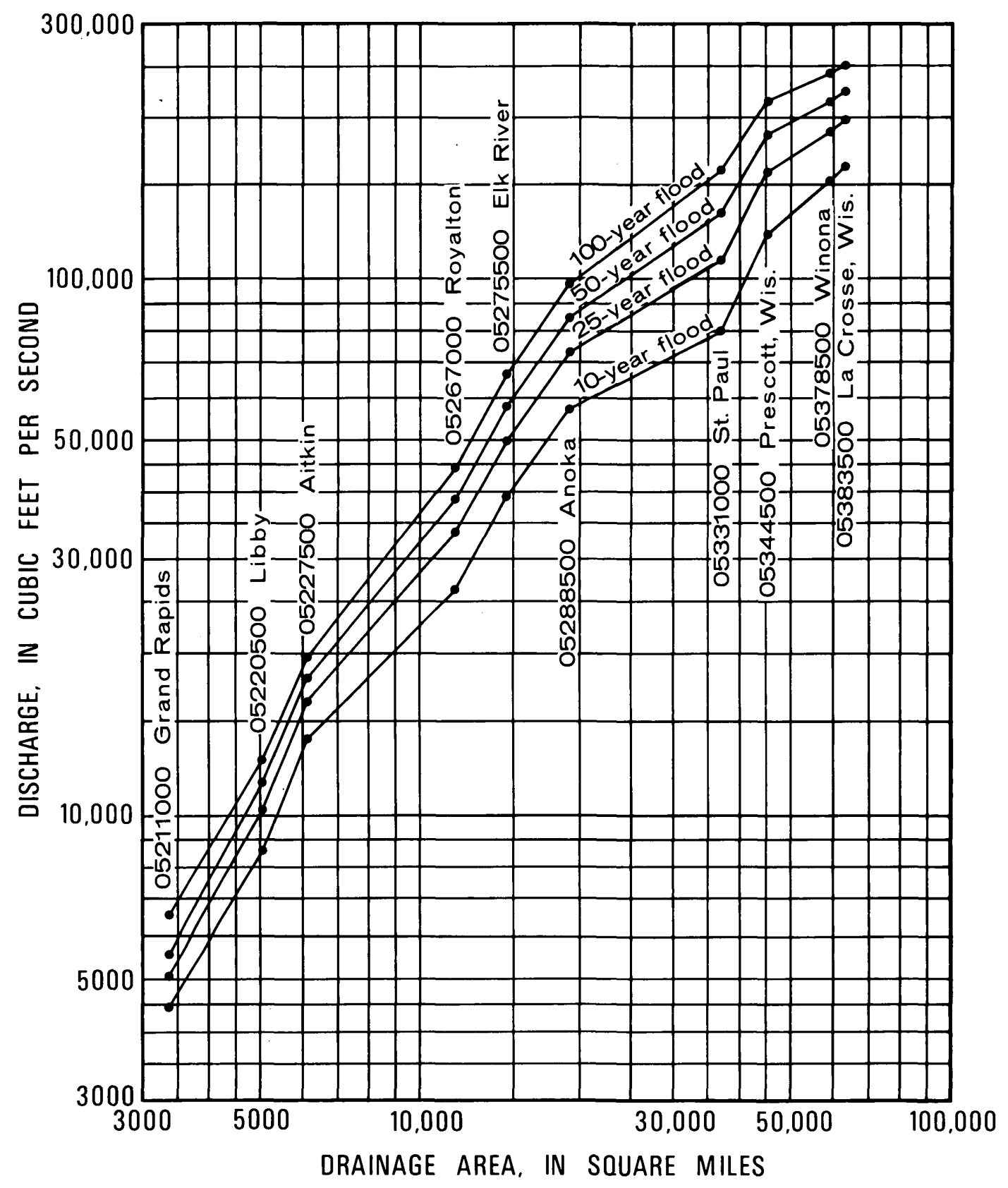

Figure 3.--Relation of flood magnitudes for selected recurrence intervals to drainage area, Mississippi River main stem. 


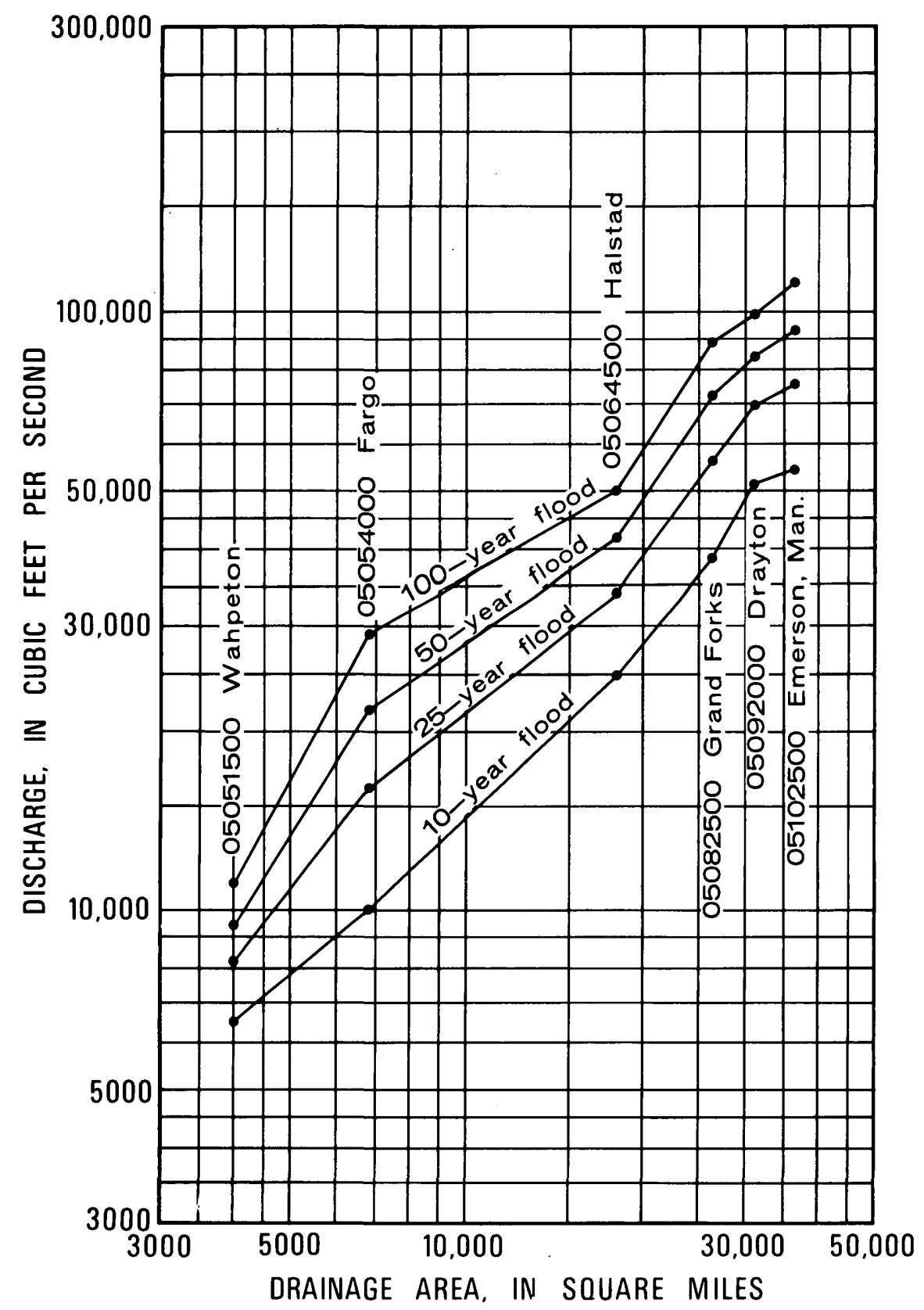

Figure 4.--Relation of flood magnitudes for selected recurrence intervals to drainage area, Red River of the North main stem. 
In general, estimates of future flood occurrences over a long time period become more reliable with greater length of record (Hardison, 1969). The standard error of estimate decreases with increasing years of available record, but at a decreasing rate. At or near gage sites (excluding main-stem streams), flood characteristics may be based on analysis of actual records collected at the site (from table 2), or may be computed from regional estimating relations. As noted previously, regional estimating relations will probably provide, more reliable results than the use of on-site gaging-station data if the period of record is short. It is recommended that use of tabulated gaging-station data for estimating flood characteristics at on-site, or by transfer to nearby locations, be restricted to those frequency relations based on more than 20 years of record.

The reliability of a regression equation may be judged by the standard error of estimate, which is a measure of the distribution of the observed data about the regression equation. The standard error, given in percent, is the range of error to be expected two-thirds of the time. That is, the difference between the computed and the observed discharge for two-thirds of the frequency estimates will be within plus or minus one standard error of estimate. Because the variables used in these analyses were expressed in logarithmic form, standard errors are larger in the positive direction. A graphical interpretation of the standard error for the 10-year frequency relation in Region $G$ is shown in figure 5. Table 4 lists the average standard errors of estimate for the defined relations in each region, except for Region F. Relations for that region were adapted from regression equations developed by Becker (1974) for the adjacent area in South Dakota.

Flood-frequency relations expressed in this report may be used to estimate magnitude and frequency of floods on most Minnesota streams. Applicability and reliability of these relationships is dependent on the basin characteristics at the site under consideration being within the range of characteristics used to define the frequency relations. The range in sampled basin parameters is large enough to allow use of the frequency relations at virtually all sites where streamflow is not significantly affected by regulation, diversion, or urbanization. Exceptions will occur in those instances where the site, for which estimates are required, falls immediately below a lake or ponding area where large storage capacity, in relation to total drainage area size, could seriously alter the outflow flood characteristics. In such cases, the frequency relations may be used as an aid in developing an inflow hydrograph for use in routing through the storage area. 


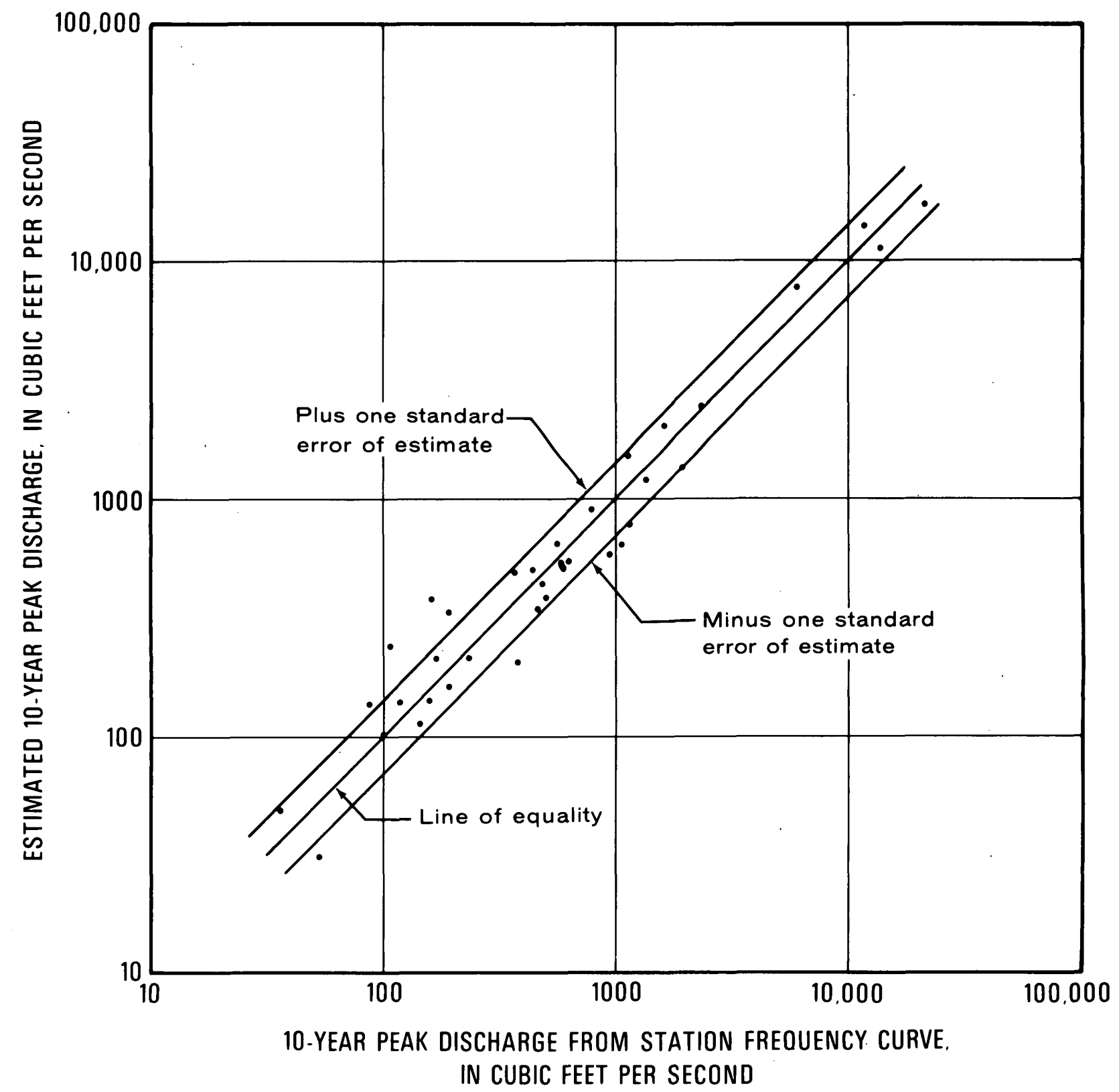

Figure 5.--Graphical interpretation of standard error of estimate for 10-year flood in Region G. 
Table 4.--Standard error of estimates for defined relations

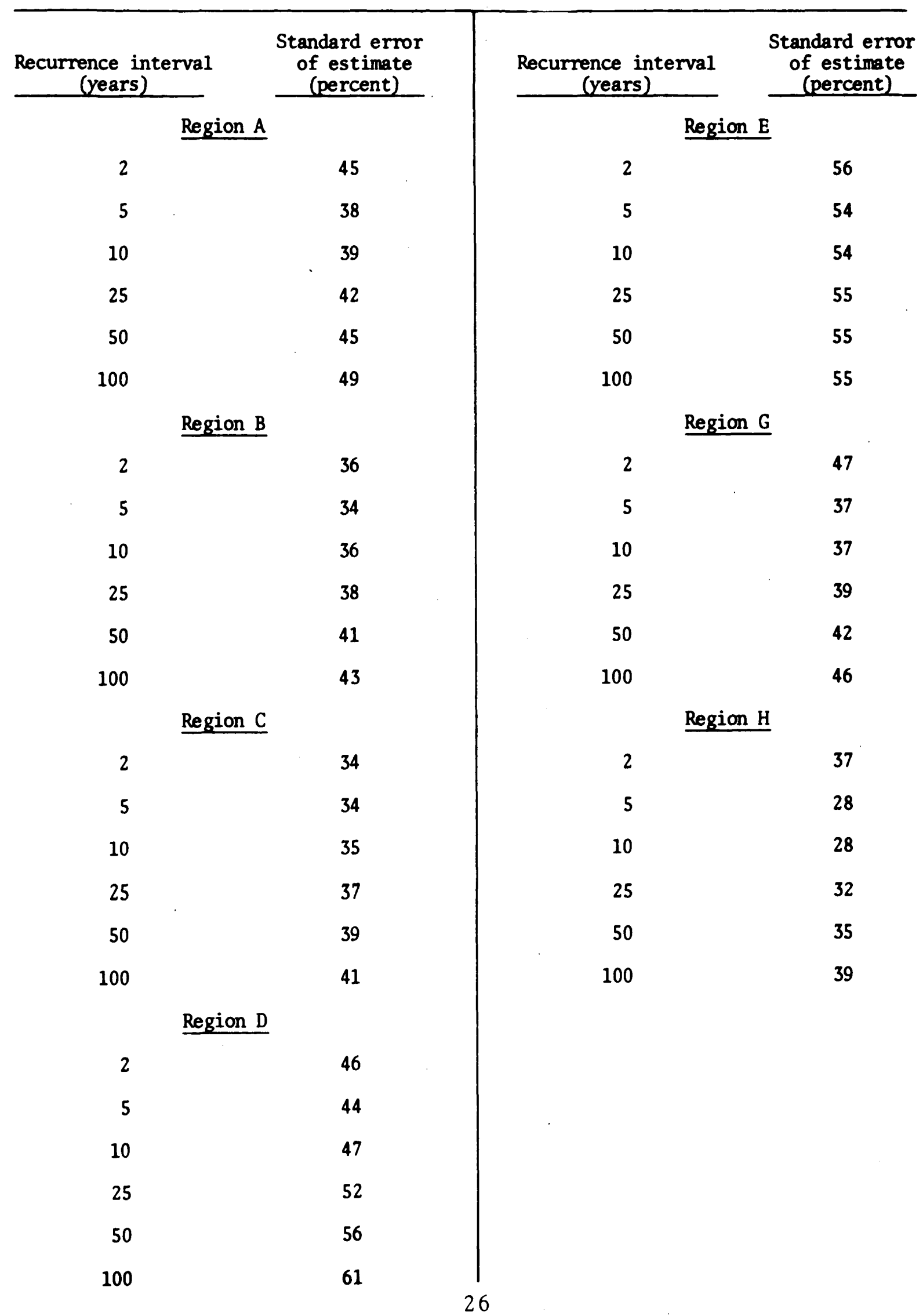


Data Used

Flood-frequency data used in the regression analysis were derived from records of 10 or more years length collected at 219 gaging stations located on natural flow streams. Stations operated by the Minnesota district consisted of 78 sites classified as continuous-record stations and 123 as partial-record stations. Records for 18 sites in adjoining states were also included. Annual peak data through the 1974 water year were considered for the Minnesota partial-record stations, through the 1972 water year for stations in adjoining states, and through the 1970 water year for Minnesota continuous-record stations. Frequency data for many of the continuous-record stations are the result of interagency coordination of 100 -year flood estimates required for numerous studies conducted in Minnesota. Where such coordination occurred, frequency curves developed by individual participating agencies were adjusted to provide a uniform peak discharge at the 100-year recurrence interval.

\section{Flow-frequency Analysis at Gaging Stations}

A flood-frequency curve for each gaging station was prepared by fitting a log-Pearson Type III frequency distribution to observed annual peaks, using regionalized coefficients of skewness. The log-Pearson Type III method is documented in U.S. Water Resources Council Bulletin No. 15 (1967).

Analysis of frequency characteristics, in connection with interagency coordination activities, had indicated a developing pattern to the variation of computed skew coefficients. LogPearson computations based on records starting in the early 1930 's generally had large negative skew values while computations based on longer term records produced skew coefficients more nearly approaching zero. Large negative coefficients of skewness were also prevalent at sites where streamflow was significantly affected by natural storage. Skew coefficients generated from records which commenced in the early 1930's, a sustained period of severe widespread drouth in Minnesota, apparently are affected to a varying degree by one or more low outliers. With extension of the record, low outliers are no longer evident.

Adoption of generalized coefficients of skewness applicable to a region was found to significantly improve the fit of the computed frequency curves as the effect of outliers (both high and low) is greatly diminished. Based on analysis of long-term 
records, adjusted plotting position of outstanding floods recorded at short-term record sites, and extension of some records by correlation, generalized skew coefficients ranging from zero to -0.2 were selected for the log-Pearson analys is of Minnesota gaging-station records.

A later publication by the Water Resources Council, Bulletin 17 (1976), recommends virtually the same procedures for log-Pearson Type III frequency analyses of gaging station records as were used in this report. Differences as they would apply to Minnesota streams are mostly in the regionalized skewness coefficient and in the treatment of historical peaks. Recommendations for generalized (regional) coefficients of skewness in Bulletin 17 range from -0.1 to -0.4 in Minnesota, which differ only slightly from the assigned skew coefficients used in this study. Historical peak adjustments would have only a minor effect on the regression analysis as historical peak data is available at only about 6-percent of the sites analyzed for this report.

Frequency analyses for each gaging station were developed by computer using standard U.S. Geological Survey programs. A computer printed plot of the frequency curve was obtained to visually compare the fit of the computed frequency curve to the observed annual peaks. Modification of the initial frequency curve, by graphical interpretation or use of a different coefficient of skewness, was made in a few instances as dictated by the available data. A sample frequency curve is shown in figure 6 .

From the frequency analyses, peak discharges for recurrence intervals of $2,5,10,25,50$ and 100 years, 1 isted as $Q_{2}, Q_{5}$, Q10, etc. in table 2 , were selected for the regression analysis.

It should be noted that changing analytical methods, interagency coordination activities, and increasing length of record will undoubtedly result in variations from the frequency estimates listed in table 2 .

\section{Multiple-regression Mode1}

Relations between peak discharge (dependent variable) and a set of basin characteristics (independent variables) were developed by multiple-regression techniques. Past experience has shown that peak discharges are linearly related to most basin characteristics if the $10 \mathrm{~g}$ transformations of the variables 


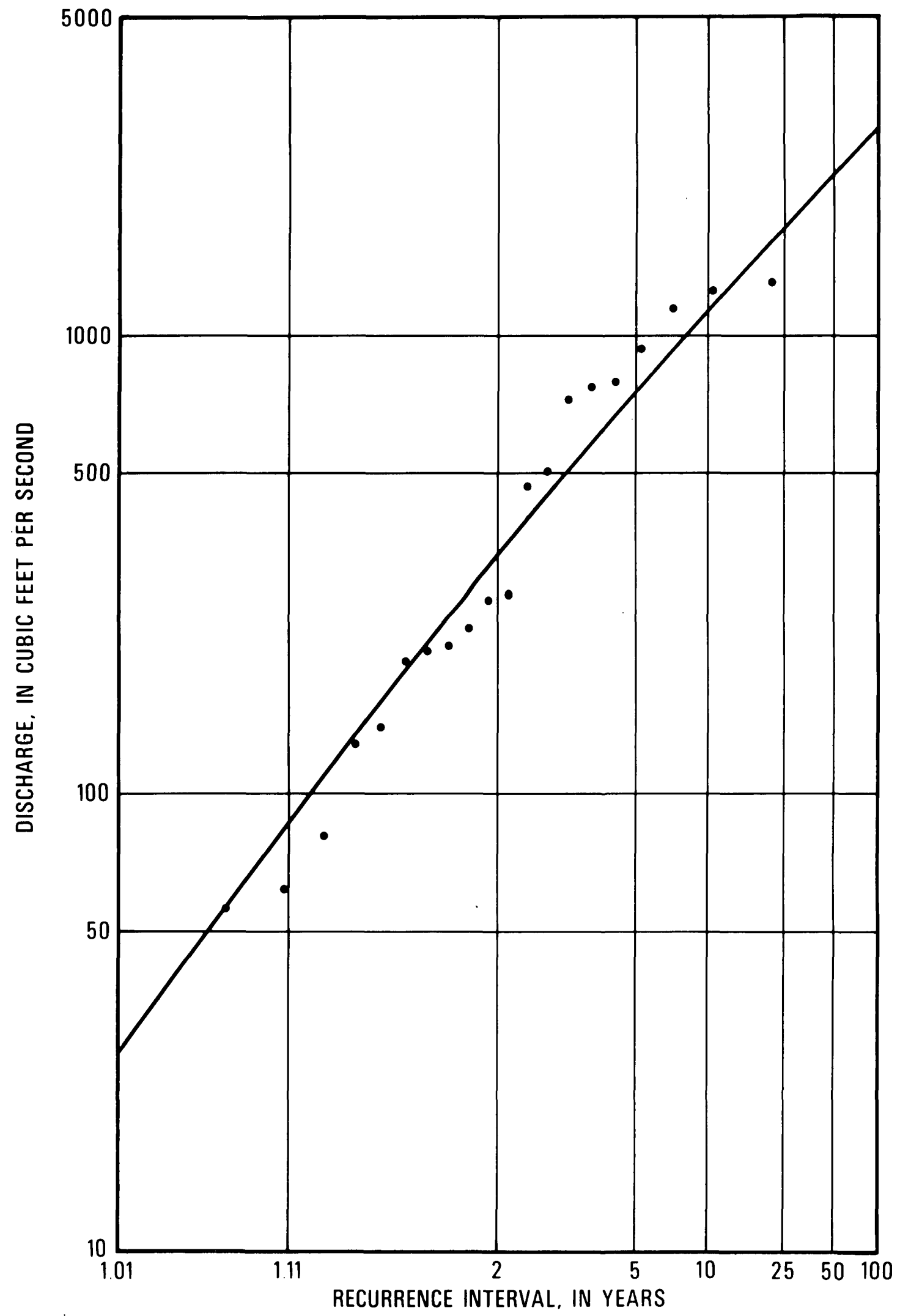

Figure 6.--Flood frequency curve for East Branch Blue Earth River near Bricelyn, Minnesota. 
are used. The regression model then is of the form:

$$
\log \mathrm{Q}_{\mathrm{T}}=\log \mathrm{a}+\mathrm{x} \log \mathrm{A}+\mathrm{y} \log \mathrm{B}+\mathrm{z} \log \mathrm{C}
$$

or it's equivalent:

$$
\mathrm{Q}_{\mathrm{T}}=\mathrm{a} \mathrm{A}^{\mathrm{x}} \mathrm{B}^{\mathrm{y}} \mathrm{C}^{\mathrm{z}}
$$

where:

$\mathrm{Q}_{\mathrm{T}}$ is the peak discharge for $\mathrm{T}$-year recurrence interval

a is a constant

$x, y$, and $z$ are regression coefficients

$A, B$, and $C$ are basin characteristics

The step-forward method of multiple-regression analysis was used wherein the regression equation is generated by adding the independent variables in the order of greatest significance to the estimating relation. The variable added for each step is the one which makes the greatest reduction in the standard error of estimate. Only those independent variables statistically significant at greater than the 95 percent confidence level are included in the equations. The analysis defines the regression constant and coefficients, standard error of estimate, and other statistical data for each frequency relation.

In a few instances, independent variables were added or deleted from the equations in order to standardize, on a regional basis, the variables to be considered at the various recurrence intervals. Original equations, in such cases, resulted in frequency curves that were irregularly shaped. Continuity of the frequency curves was vastly improved by addition or deletion of such variables with little effect on the standard error of estimate.

Hydrologic regions shown on figure 1 were defined by plotting residual values on a map. The residual errors from the regression analysis are the differences between observed and computed flood magnitudes and can be expressed in terms of ratios. The plot of residual ratios illustrated the geographical bias inherent in statewide frequency relations. The 8 regions were delineated by removal of successive areas from the regression, based on groupings of the residual values. As each area was removed, new regression relations were computed utilizing the remaining input data, and the residuals again plotted to define the remaining areal bias. 
Inter-regional comparisons of the regression equations were made by computing flood magnitudes, at selected recurrence intervals, using constant basin characteristics for each regional relation. These tests showed considerable variability in computed flood characteristics across the State for a fixed set of independent variables.

Regional boundaries outlined on figure 1 generally follow basin divides. The following exceptions based on topographic or geologic features are: 1) in the southwestern part of the State, where the regional boundary follows the break in slope along the Coteau des Prairie and crosses the upper end of the Redwood, Yellow Medicine and Lac qui Parle River basins; and 2 ) in the northwestern part, where the regional boundary follows an upper beach ridge of glacial Lake Agassiz and crosses the upper end of the Wild Rice, Marsh, Sand Hill, and Clearwater River basins.

\section{Basin Characteristics Investigated}

A precondition established for this report was that the number of basin characteristics used in the regression be limited in number, and be readily determined from available maps, to eliminate the necessity for on-site measurements. Independent variables investigated in addition to area, slope and storage, were forest cover and soil type. Forest cover index was used as the percentage of the drainage area covered by forests increased by 1 percent. The soil index was determined by averaging residuals from an initial regression according to different soil types. The soil index was tested separately on a multiple regression analysis of small basins where soil homogeneity was more probable. Neither basin characteristic improved the estimating relations.

\section{SUMMARY}

Basin characteristics and peak flows for 2-, 5-, 10-, 25-, $50-$, and 100-year recurrence intervals were tabulated for 201 gaging stations ranging in size from 0.05 to $5,280 \mathrm{mi}^{2}$. The data from these 201 stations were grouped according to eight distinct hydrologic regions and equations were developed for each region by relating peak flows to basin characteristics. The resulting equations provide a method for estimating floodfrequency relations for ungaged sites on all natural streams. In addition, graphs are presented for determining floods of selected recurrence intervals for large streams which may be significantly affected by regulation. 
The equations developed by multiple regression for estimating peak flows at ungaged sites determined that drainage area, slope, and storage are significant basin parameters for estimating 2-, 5-, 10-, 25-,50-, and 100-year floods. Drainage area, slope, and storage were significant in 4 of the 8 regions, drainage area and slope in 2, and only drainage area in the remaining 2 regions. Accuracy of estimates obtained by using the equations are discussed and the standard error of estimate is given for each equation. Standard errors ranged from 28 to 61 percent.

Analytical techniques are discussed for determining flow frequency at gaging stations and for making estimates at ungaged sites. The regional regression equations provide more reliable results than frequency relations defined for gaging stations if the period of record is short. For sites at or near gaging stations having 20 or more years of record, the tabulated station data are considered more accurate than estimates from the regression equations. 


\section{REFERENCES}

Becker, L. D., 1974, A method for estimating magnitude and frequency of floods in South Dakota: U.S. Geol. Survey Water-Resources Inv. 35-74, 78 p.

Hardison, C. H., 1969, Accuracy of streamflow characteristics: in Geological Survey Research, U.S. Geol. Survey Prof. Paper 650-D, p. D210-214.

Patterson, J. L., and Gamble, C. R., 1968, Magnitude and frequency of floods in the United States, Part 5: U.S. Geol. Survey Water-Supply Paper 1678, 546 p.

Prior, C. H., 1949, Magnitude and frequency of floods in Minnesota: Minnesota Dept. of Conservation, Div. of Waters Bull. $1,128 \mathrm{p}$.

Prior, C. H., and Hess, J. H., 1961, Floods in Minnesota, magnitude and frequency: Minnesota Dept. of Conservation, Div. of Waters Bull. 12, 142 p.

Thomas, D. M., and Benson, M. A., 1970, Generalization of streamflow characteristics from drainage-basin characteristics: U.S. Geo1. Survey Water-Supply Paper 1975, 55 p.

U.S. Water Resources Council, 1967, A uniform technique for determining flood flow frequencies: Hydrology Committee Bu11. 15, $15 \mathrm{p}$.

1976, Guidelines for determining flood flow frequency: Hydrology Committee Bul1. 17, $196 \mathrm{p}$.

Wiitala, S. W., 1965, Magnitude and frequency of floods in the United States, Part 4: U.S. Geol. Survey Water-Supply Paper $1677,357 \mathrm{p}$.

1971, Red River of the North regional flood analysis: North Dakota State Water Commission and Minnesota Dept. of Natural Resources, $19 \mathrm{p}$.

1973, St. Croix River regional flood analysis (St. Croix Fal1s, Wis. to mouth): Minnesota Dept. of Natural Resources and Wisconsin Dept. of Natural Resources, 19 p. 


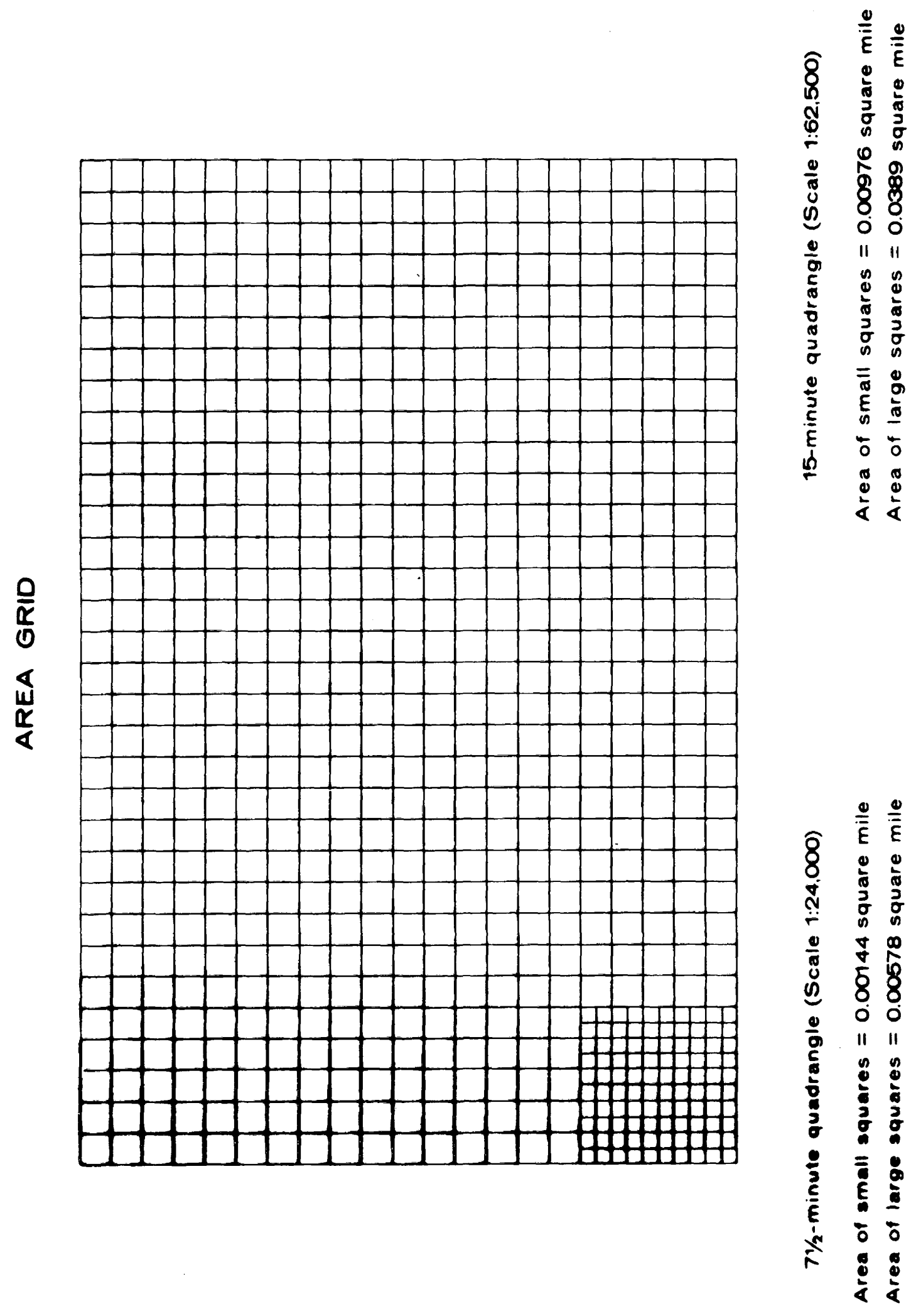

